Check for updates

Cite this: RSC Adv., 2017, 7, 32164

\title{
Progress and perspectives in bioactive agent delivery via electrospun vascular grafts
}

Received 27th April 2017 Accepted 8th June 2017

DOI: 10.1039/c7ra04735e

rsc.li/rsc-advances

\begin{abstract}
Marek Rychter, (D)*ab Anna Baranowska-Korczyc (D) ${ }^{\mathrm{b}}$ and Janina Lulek ${ }^{\mathrm{a}}$
The review discusses the progress in the design and synthesis of bioactive agents incorporated into vascular grafts obtained by the electrospinning process. Electrospun fibers can be applied as an artificial extracellular matrix for tissue engineering and drug delivery, improving tissue regeneration and therapeutic outcomes. A large number of active substances are loaded into the fibers, such as growth factors, heparin, NO donors, statins, antibiotics or anti-inflammatory agents. There are various methods for bioactive substance incorporation including direct blending, emulsion, and coaxial electrospinning as well as covalent and non-covalent binding of the bioactive molecules to the fiber surface. The release mechanism of the drug depends on the synthesis route, active substance properties, and polymer matrix nature. The electrospun materials represent a very promising building block for the fabrication of effective vascular prosthesis in the near future.
\end{abstract}

\section{Introduction}

Cardiovascular diseases are still the leading cause of death in developed countries, accounting for more lives each year than cancer and chronic lower respiratory disease combined. If current tendencies continue the number of cases will increase to 25 million in $2020 .{ }^{1}$ Atherosclerosis of blood vessels which is a common denominator of various cardiovascular diseases results in the pathological thickening of their lining with subsequent plaque formation and is considered to be the leading cause of sudden deaths in the USA. ${ }^{1}$ Despite extraordinary progress made in the field of atherosclerosis prevention and treatment involving new thrombolytic agents and greater accessibility to modern less-invasive angioplasty procedures, a significant number of coronary artery diseases and peripheral vascular diseases still require vascular grafts. The currently available options for vascular grafts ${ }^{2}$ have specific disadvantages including limited availability of suitable donors for autologous transplantations, ${ }^{3}$ inflammation or calcification of xenografts ${ }^{4}$ and finally thrombosis and neointimal hyperplasia of synthetic non-degradable prostheses. ${ }^{5}$

In recent years, tissue-engineered vascular grafts capable of inducing neovascularization process have gained huge interest. $^{6-8}$ Several reports related to scaffold-free grafts and their formation from biodegradable materials with high compatibility and similarity to surrounding and autologous tissue have been noted. ${ }^{9-11}$ To design effective and efficient

${ }^{a}$ Department of Pharmaceutical Technology, Faculty of Pharmacy, Poznan University of Medical Sciences, Grunwaldzka 6, 60-780 Poznan, Poland. E-mail: mrychter@ump. edu.pl

${ }^{b}$ NanoBioMedical Center, Adam Mickiewicz University Poznan, Umultowska 85, 61614 Poznan, Poland polymer-based vascular grafts, their properties should be similar to natural blood vessels, including thrombotic resistance and sufficient mechanical strength. Additionally, they need to be capable of inducing endothelial coverage, followed by gradual degradation till total resorption with full tissue regeneration. ${ }^{12}$ Moreover, materials involved in graft fabrication should be non-immunogenic and should demonstrate properties promoting cell attachment and growth. Nevertheless, still there are certain unsolved issues connected with scaffold-based systems for vascular tissue engineering, such as lack of sufficient growth factor reservoir ${ }^{13}$ or improper cell recruitment, differentiation, and proliferation caused by the influence of scaffold nanotopography. ${ }^{14}$

In order to solve the problem of insufficient revascularization, occurring after graft implantation, a number of studies are currently focused on integrative approach involving the use of polymer scaffold as bioactive substance delivery system. The drugs are incorporated into the polymer matrix or immobilized on the outermost surface to these scaffolds. ${ }^{15}$ Polymer-based scaffolds demonstrate characteristics of advanced drug delivery systems for cardiovascular diseases treatment, including high loading capacity, high loading efficiency of single and multiple active substances. Moreover, they prevent fast release and rapid clearance of labile compounds e.g. growth factors, which significantly improve their delivery profile. ${ }^{16}$ It was found that the natural, as well as synthetic compounds, have demonstrated the ability to originate revascularization process. Advanced scaffolds have combined advantages of synthetic polymers including high mechanical durability and high resistance to processing conditions with an abundance of cell binding sites of natural polymers in order to improve overall biocompatibility. ${ }^{7,17}$ 
This paper reviews the synthesis and properties of polymerbased scaffolds and effects of their use as bioactive substance delivery materials in vascular tissue engineering. The discussed data are focused on the electrospinning process and its considerable interest in vascular tissue engineering. The study provides new insights into electrospun materials and their application as bioactive substance delivery base with a special attention payed to the treatment of cardiovascular conditions. In this contribution, electrospinning is presented as the most effective, efficient and low-cost method for vascular graft fabrication and development. Additionally, several techniques of active substance incorporation and immobilization to electrospun materials are also provided. The release mechanism of the active substance from polymer-based scaffolds is also presented. This review indicates that polymer electrospun fiber materials offer a great potential as vascular grafts for tissue engineering.

\section{Electrospinning of vascular grafts}

Several reports have been published on different approaches for fabrication of biodegradable vascular grafts and polymer drug delivery systems. These investigations describe electrospinning, molecular self-assembly, hydrogels, thermally induced phase separation and solvent casting-particulate leaching techniques. ${ }^{7}$ The most promising one is electrospinning due to the formation of quasi one-dimensional nano- and micro structures, direct control of obtained structure morphology and overall efficiency of the procedure.

Electrospinning process allows to obtain the fibers characterized by a different diameter, from nano- to micro-meters, controlled by process parameters. This method can produce composite fibers depending on the formulation of starting polymer solution. ${ }^{\mathbf{1 8}}$ However, synthetic polymer degradation time should be appropriate for cell infiltration and tissue restoration guided by electrospun vascular graft. ${ }^{19}$ The natural polymers, such as collagen, elastin or gelatin, are widely used as an additional component to increase cell attachment. ${ }^{20}$

During electrospinning process, the syringe containing polymer solution is connected to a syringe pump, which is responsible for a constant and controllable fed rate. Fig. 1 presents a scheme of a typical electrospinning setup with a rotating drum collector. In general, the mechanism behind electrospinning technique is based on the electrostatic force, which is used to stretch the solution ejected from a spinneret. When a high voltage is applied to a metallic needle, a repulsive force is formed, as the induced charges are evenly distributed over the surface of the polymer drop, which is coming out from the metallic needle. When the electrostatic forces exceed the surface tension of the viscoelastic solution, the jet bursts from the spinneret. As the elongation phase continues the location of charge in formed jet starts to change. This process of continuous bending causes the jet to become unstable which results in a number of bending instabilities, which play a crucial role in the reduction of jet diameter. ${ }^{21}$ In an electric field the solvent evaporates during jet stretching process and finally, solutes solidify on the collector. ${ }^{22}$
In order to dissolve the polymers for electrospinning process, different organic solvents are used. However, solvents are only utilized for manufacturing purposes. Therefore, in the case of electrospun cell scaffolds they should be removed, to the highest extent possible to limit the potential toxicity of their residues. The safety data for residual solvent levels are presented in Pharmacopeia ${ }^{23}$ and based on this data they are divided into three classes. In the case of the electrospinning technique, the most desirable organic solvents which can be used for tissue engineering purposes are classified to class 2 or class 3 which both are characterized by a limited toxicity.

The electrospinning is a highly modifiable technique for which a number of different electrospinning set-ups were developed including a rotating drum collector, which is applied to tubular structures preparation used as vascular grafts (Fig. 1). This approach provides an efficient solution for producing tubular structures of various sizes. The external diameter of drum collector corresponds to the internal diameter of produced structure. However, methods of electrospun material detachment from rotating drum collector were rarely reported in detail. Some insightful comments on different reported approaches were described by Errico et al. which could be helpful to develop a suitable methodology for tubular structure production for vascular grafts application. ${ }^{24}$

One of the advantages of a rotating drum collector is the fact that it is capable of orienting fibers circumferentially which is crucial in the development of electrospun vascular graft. Aligned fibers instead of randomly oriented ones are also obtained by either varying the rotating collector parameters or applying additional magnetic or electric field frames. ${ }^{21}$ Uniaxial aligned fibers have anisotropic tensile properties, which redistributes tensile force equally over produced structure, which would be vital for developing vascular grafts which need to withstand high pressure after their implantation. ${ }^{25}$ Fibrous scaffolds produced by electrospinning, not only have directional mechanical properties but also can facilitate the orientation and attachment of cells to the fibers. The structures obtained by electrospinning technique have a great potential in mimicking the extracellular matrix (ECM), ${ }^{26}$ because produced fibers are in the size range of ECM present in natural tissues of living organisms.

Process parameter like higher collector rotation speeds could contribute to greater uniformity in fibers diameter and thus more homogenous mechanical properties of single fibers which have implications in tissue regeneration at the cellular level. As the cell in vivo requires mechanical interaction with ECM to achieve its physiological function like differentiation or tissue restoration, improved mechanical equilibrium of artificial vascular graft will be supportive in neo-tissue formation. Therefore, it is important to consider nano- or micromechanical properties of vascular grafts produced by rotating drum collector, due to their impact on cell function and tissue restoration.

Additionally, electrospun fibrous vascular grafts have high surface area and high loading capability which expand their use into drug delivery systems. Furthermore, the arrangement of fibers protects the incorporated bioactive molecules, provides 


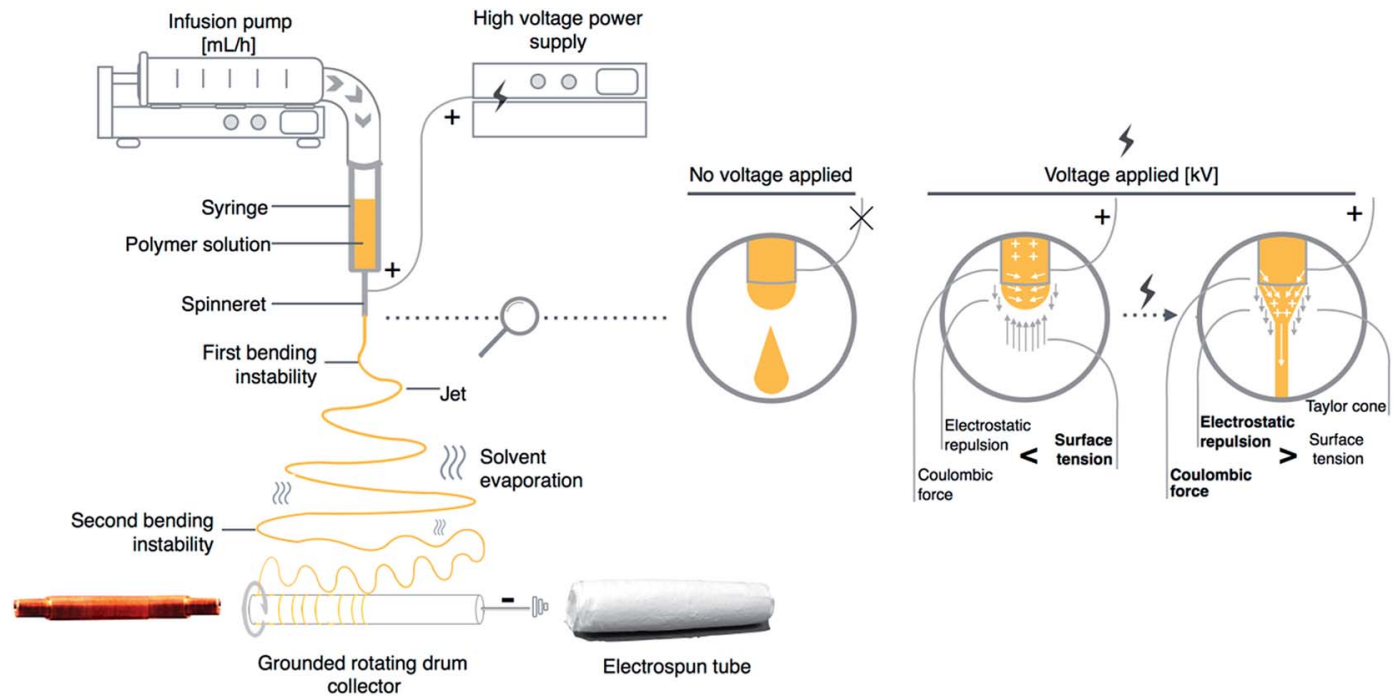

Fig. 1 Scheme of basic electrospinning setup for vascular graft preparation with a graphic representation of the effect of high voltage on electrospun polymer solution.

means for their localized delivery and simultaneously limits the general side effects of traditional drug administration.

\section{Incorporation of bioactive substance to electrospun vascular grafts}

The electrospun materials exhibit specific and novel features, which make them interesting as potential delivery systems. Large surface area to volume ratio and high porosity helps to overcome the problem of bioactive substance loading to currently used combination products like for example drugeluting metal stents. Additionally, this approach improves and regulates the dissolution of the bioactive substance, which could be controlled by the nano- and micro-structure of the electrospun polymer fibers. In recent years several drugs have been incorporated into electrospun materials including antibiotics $^{27,28}$ and anticancer drugs ${ }^{29-31}$ proving the feasibility of this approach. In this part, we will focus our attention on the methods of bioactive substances loading into electrospun vascular grafts. There are various techniques and set-up modifications used to adsorb, immobilize or incorporate bioactive substance to electrospun vascular grafts (Fig. 2). Examples of application of each discussed incorporation technique to obtain vascular grafts loaded with bioactive substance are presented in Tables 1 and 2.

\subsection{Non-covalent immobilization}

Non-covalent immobilization is the most convenient procedure of binding the bioactive molecules to the fiber surface. This

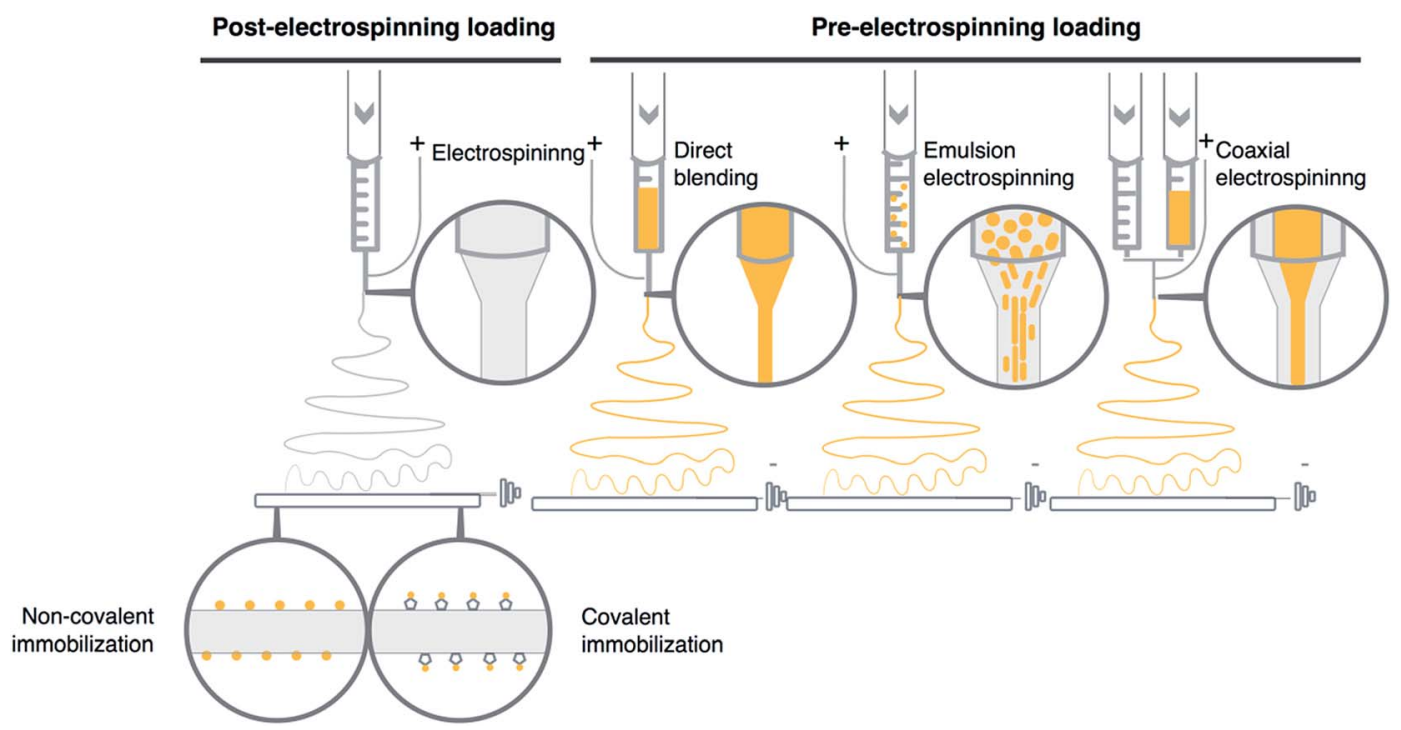

Fig. 2 Bioactive substance incorporation and immobilization methods to electrospun materials. 




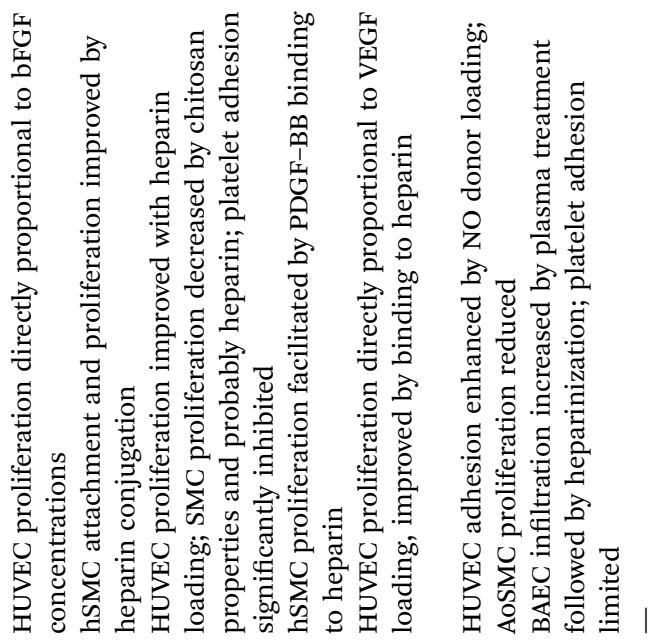

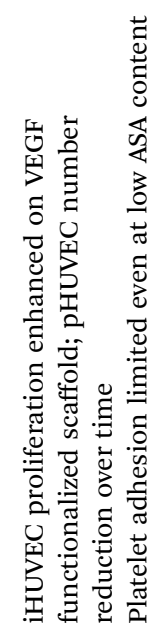
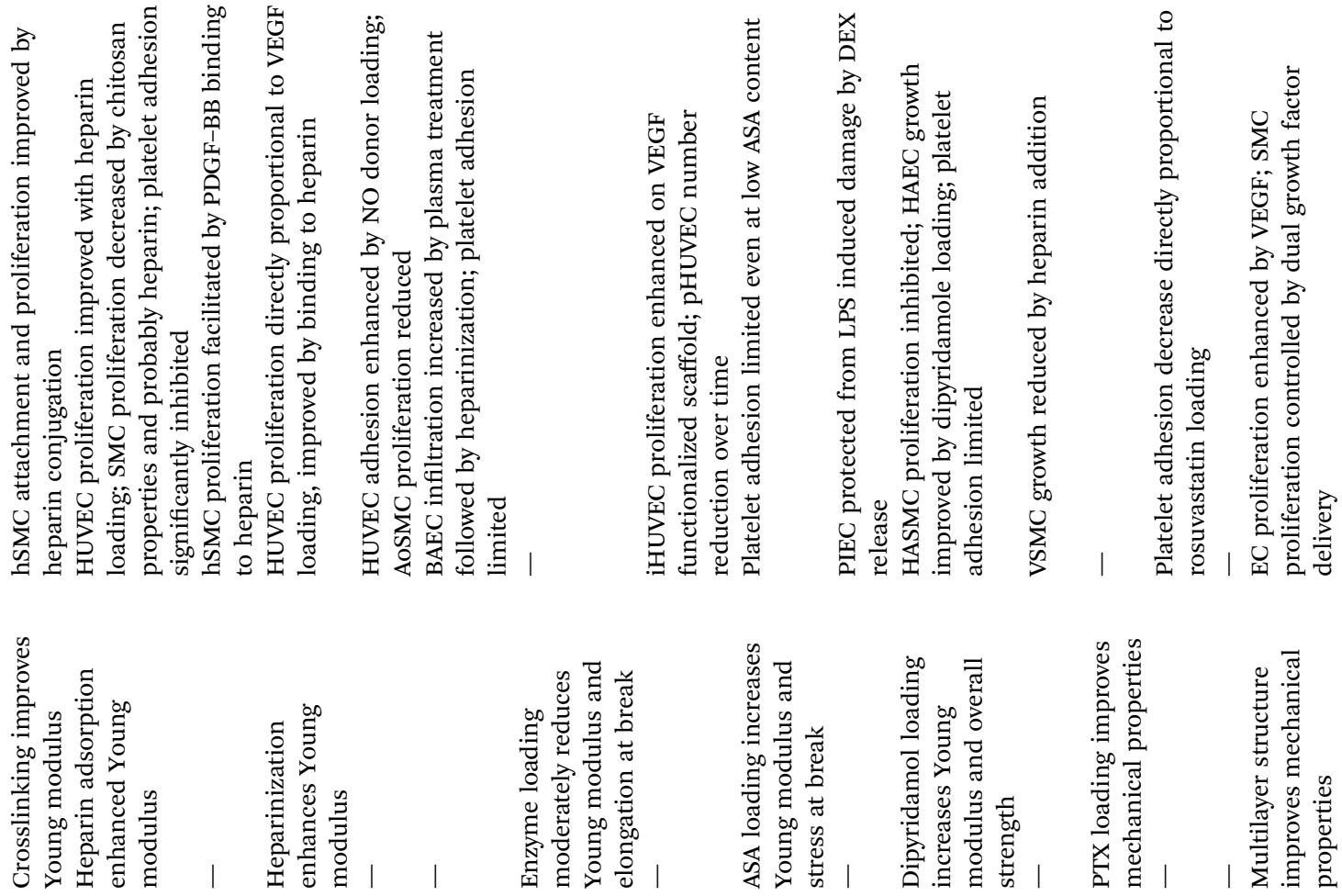

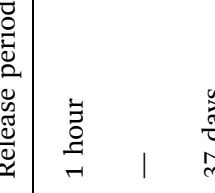

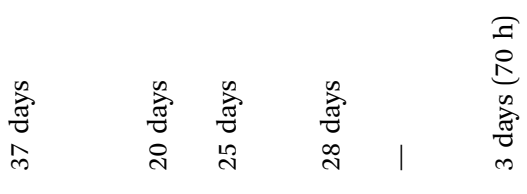

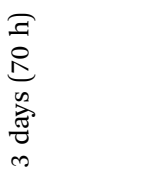

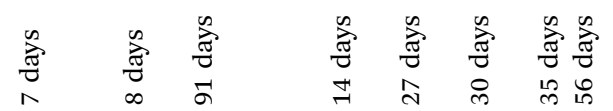



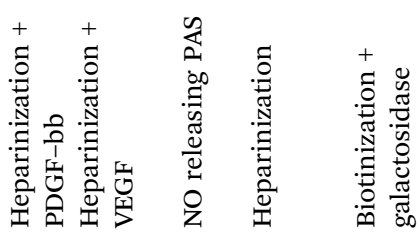

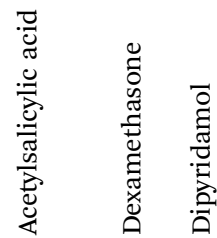

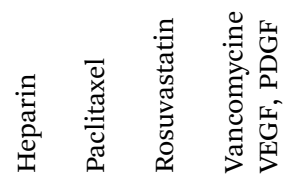

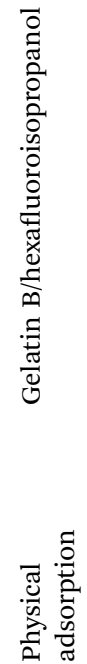




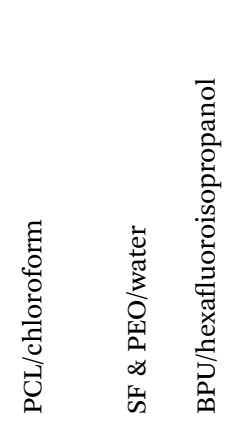

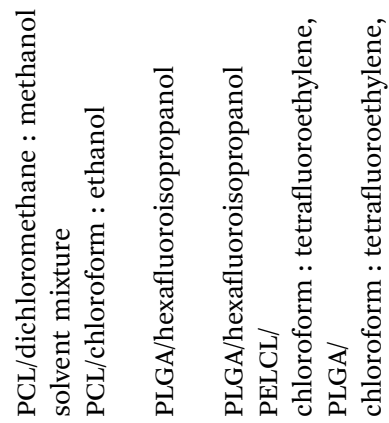


post-processing method is based on a simple process, where the electrospun graft is immersed in a solution containing the bioactive substance (Fig. 2). Electrospun grafts have large surface area due to a high number of nano and microfibers which can adsorb different active compounds on its external surface.

This approach is characterized by relatively mild conditions of the procedure which make it a perfect solution for labile compounds immobilization including proteins like for example growth factors used to improve vascular tissue restoration (Tables 1 and 2). Despite a number of advantages, the non-covalent binding technique is also characterized by a rapid dissolution of bioactive substance from polymer fibers and poor control over its release kinetics. However, in the case of hydrophilic substances like growth factors, which often cause burst release, various methods of surface modification were developed. One of the most interesting is fiber surface heparinization. In the first step, polymer graft surface is usually treated with plasma in order to increase hydrophilicity and activate surface for further modification of amine groups. ${ }^{32,33}$ After the surface functionalization with heparin, improved affinity towards various growth factors is used to adsorb bioactive molecules via electrostatic interaction on the fiber surface. No-plasma treatment methods were also developed. In such case, electrospun fibers underwent cross-linking procedures which also resulted in free amine groups on fibers surface. ${ }^{34}$

\subsection{Covalent immobilization}

Due to successful covalent immobilization of heparin on the surface of electrospun fibers, this method was also applied for efficient immobilization of other bioactive molecules (Fig. 2). Similarly, to non-covalent immobilization, it is a postelectrospinning process. One of the greatest advantages of this approach is the nature of covalent bonding itself, which provides better long-term stability of these modifications as well as improved efficacy of the whole process. The covalent immobilization demonstrates high loading capacity and more sustained drug release profile of bioactive molecules. Despite mentioned advantages, this form of bioactive substance bonding to the surface of the fibers makes it relatively challenging, especially from the practical point of view. As the modification process involves a number of steps, this makes eventual scale-up process of the whole method more difficult. Furthermore, more complex modifications involve a high risk of possible changes in physical and especially mechanical properties of electrospun graft, which are essential for cardiovascular application, in which these structures need to withstand pressures and forces encountered in in vivo conditions. ${ }^{35}$ Additionally, functional groups formation on the fiber surface utilizes substances and conditions, which increases the risk of the decomposition of labile bioactive substances. Nevertheless, some groups have managed to successfully immobilize vascular endothelial growth factor (VEGF) on electrospun scaffolds through chemical bonding, which did not affect properties of this bioactive compound. ${ }^{36}$ Other examples of bioactive substance loaded vascular grafts obtained by this technique are presented in Tables 1 and 2. 


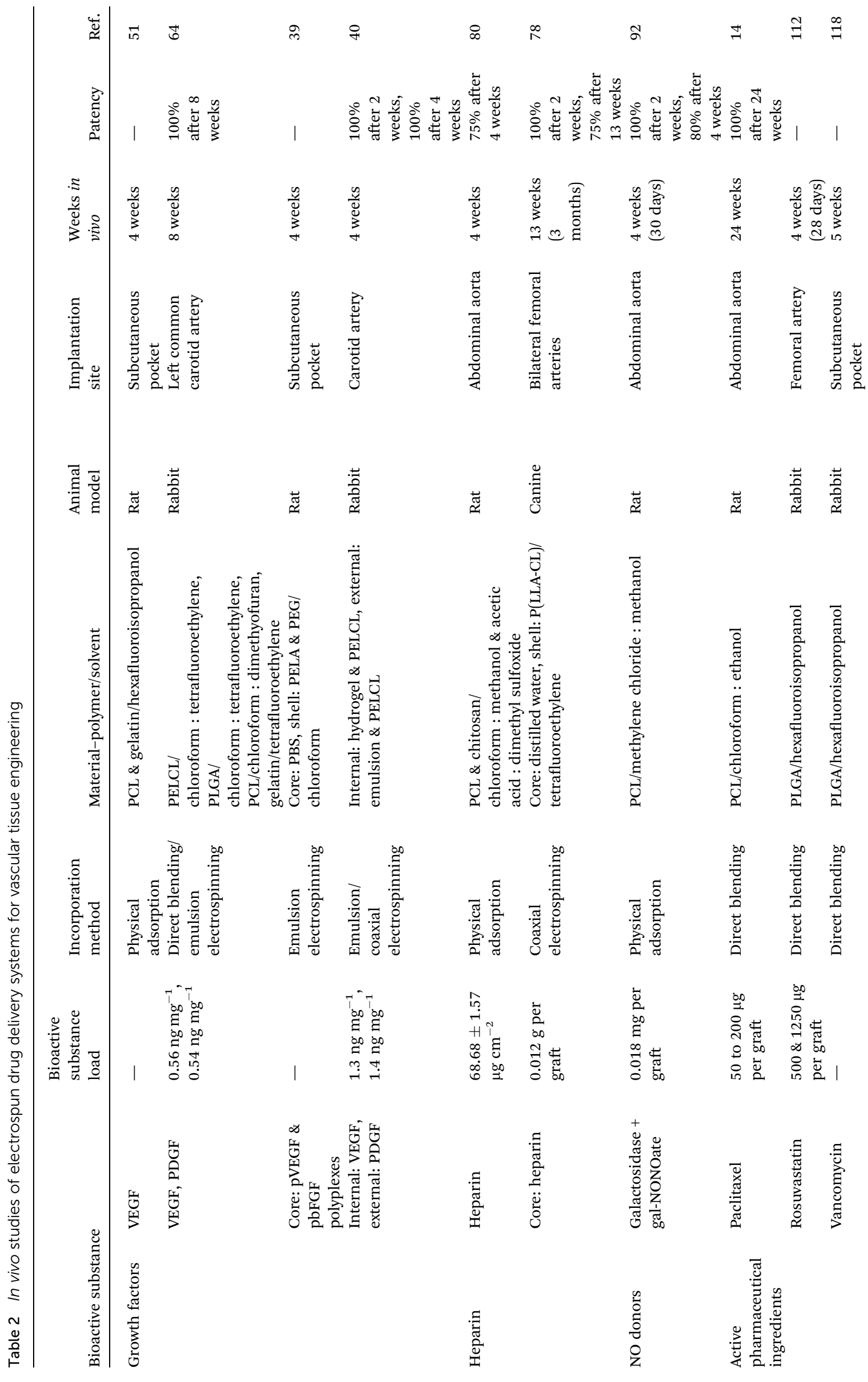




\subsection{Direct blending}

In direct blending technique, the active substances are suspended or dissolved in polymer solution shortly before the electrospinning process (Fig. 2). This technique is applied to limit the risk of active substance decomposition.

Direct blending is an optimal solution when bioactive substance and polymer show similar solubility in the solvent. However, the problem of varying solubility might be addressed by dissolving the bioactive substance in solvent miscible with polymer solution. ${ }^{37}$

In the case of similar solubility in organic solvent, the bioactive substance usually is incorporated directly into the structure of electrospun fibers. Depending on the relation between graft composition and porosity, the release profile may be easily tuned. Perhaps the most serious disadvantage of this incorporation technique involves a high risk of burst release most often caused by bioactive substance location on the surface or near the surface of electrospun fibers. ${ }^{27,38}$ This method is frequently applied for bioactive substance loading to electrospun vascular grafts (Tables 1 and 2).

\subsection{Emulsion electrospinning}

Emulsion electrospinning is one of the most efficient and novel techniques used for bioactive substance incorporation into electrospun vascular grafts (Fig. 2). There are two types of emulsion oil in water $(\mathrm{O} / \mathrm{W})$ and water in oil $(\mathrm{W} / \mathrm{O})$ which can be electrospun. However, W/O emulsions are the most optimal ones for vascular grafts preparation, due to the limited hydrophilicity of the external part of fibers which is more favorable in conditions found in living organisms. ${ }^{39}$ The electrospun solutions usually contain hydrophobic phase with the polymer dissolved in an organic solvent and dispersed hydrophilic phase containing the bioactive substance. The hydrophobic phase enables the incorporation of bioactive substances due to their solubility and dispersibility in organic solvents applied for electrospinning process.

When the high voltage is applied fibers with vesicles containing dispersed part of the emulsion in more easily solidifying dispersion medium can be formed. Core-shell fibers can be also formed as a result of relatively fast evaporation of solvent from the region close to the surface in comparison to the central part of the polymer jet. In such circumstances, the viscosity gradient of the core and shell segments changes dramatically and causes a slower rate of the evaporation process in the central part of the jet. In both cases solidified polymer acts as a hydrophobic barrier which provides protection from external conditions for labile bioactive substances including proteins. It was shown during in vitro experiments that burst release effect was limited by applying emulsion electrospinning, therefore it could potentially limit leaching-out effect of shear stress encountered in vivo by bioactive substance loaded vascular grafts. ${ }^{\mathbf{4 0 - 4 2}}$ Examples of its use in vascular graft development are presented in Tables 1 and 2.

\subsection{Coaxial electrospinning}

One of the significant breakthroughs in the case of electrospinning technique was an introduction of a coaxial spinneret.
Unlike standard spinneret, a coaxial one contains core-shell nozzle which is connected to a double compartmented syringe (Fig. 2), two separate pumps and pipelines. As a result, the described setup is capable of electrospinning two different polymer solutions at different rates, which form core-shell fibers.

This technique enables the synthesis of the fibers made from substances not usually applied for electrospinning process of vascular grafts. ${ }^{43}$ The core-shell structures are usually similar to the fibers which could be obtained by emulsion electrospinning, where the hydrophobic shell covers and protects the bioactive substances located in the hydrophilic core.

This design improvement reduces burst effect and allows dual-drug loading to both core and shell layers. ${ }^{19}$ The contact of bioactive substances and organic solvents used in electrospinning is significantly reduced which makes this method more valuable option for labile substance processing (Tables 1 and 2). Moreover, core and shell diameters of the fibers can be controlled and design by changing the process parameters including the flow rate and applied voltage. Coaxial electrospinning reveals better processing versatility compared with emulsion electrospinning or any other form of bioactive substance incorporation method. ${ }^{\mathbf{4 0 , 4 4}}$

\section{Release mechanism}

In general, the bioactive substances are transferred into vessel lumen by diffusion and medium-driven flow from the surface of fibers or from the polymer matrix which builds each electrospun fiber of vascular graft. Diffusion is the main release mechanism of bioactive substance for most biodegradable polymeric fibers. However, the biodegradability of the polymer matrix also influences the release profile, which is important for designing materials for tissue engineering application.

The diffusion from polymeric fibers occurs through direct release from the polymer matrix and diffusion across a barrier formed by polymer itself. ${ }^{45}$ In this diffusion-based mechanism, the release process of molecularly dispersed bioactive substances from the electrospun graft includes passage of bioactive substance molecules through pores within polymer matrix and passage between polymer chains. Both types of diffusion are influenced by the structure of a single fiber and will be the dominant release mechanism in the early stages after vascular graft implantation. The rate of diffusion depends on various factors including crystallinity, physicochemical properties, partition coefficient, the amount of bioactive substance loaded, fiber diameter and its structure.

Most of the polymers used in electrospinning are in semicrystalline state and form amorphous regions during the spinning process. Bioactive molecules are usually located in the amorphous regions of the semi-crystalline polymers and the initial drug release occurs mostly from these regions of the fibers. ${ }^{37}$ An increase in crystallinity results in a decrease in the diffusion rate of the bioactive substance. It is attributed to the water penetration of semi-crystalline polymers which initially occurs in the amorphous regions. ${ }^{46}$ The water infusion from the 
amorphous regions facilitates the release process and increases the diffusion rate.

The release of bioactive substances from electrospun constructs depends on substrate properties therefore when designing bioactive substance loaded vascular graft both physical and chemical properties of the active substance ${ }^{47}$ and polymer, ${ }^{48}$ must be considered.

Hydrophobic substances loaded to electrospun grafts show lower tendency to be distributed in the blood and therefore have slower clearance as their diffusion rate is slow. Contrary, hydrophilic substances have a higher attraction towards aqueous medium, therefore, their clearance is high whereas retention within graft is limited due to fast diffusion rate. In the case of polymer or polymer blends, their hydrophilicity will influence the wettability and aqueous medium penetration through the whole structure.

Another important issue closely related to physicochemical properties of the polymer and bioactive substances is partition coefficient. ${ }^{31,36}$ The partition coefficient of bioactive substance and polymer can influence bioactive substance distribution within the polymer matrix or near the surface of electrospun fibers. Therefore, it can significantly influence the release rate after graft implantation leading to a frequently encountered problem of initial fast release phase also known as a burst effect. It was found that for some polymer systems the first stage of bioactive substance release is very fast and is responsible for a rapid release of a large part of incorporated dosage. ${ }^{\mathbf{4 0 - 4 2}}$ Additionally, it was found that higher drug loading contributes to a higher risk of burst effect. Therefore, a large part of loaded dosage which rapidly diffuses from the vascular graft may cause severe local toxicity shortly after graft implantation and therefore formulations with high drug loadings should be developed with caution. The initial fast release phase is also typical for fibers loaded with bioactive substance via post-electrospinning incorporation techniques where fast desorption process from the fiber surface drives a rapid depletion of bioactive substance loading. .49,50 $^{\mathbf{4}}$ However, covalent immobilization in comparison to non-covalent methods of bioactive substance immobilization, significantly limits the burst release effect. $^{51}$ In direct blending technique, a high voltage applied during electrospinning process influences the homogenous polymer mixture in the spinneret and can locate evenly distributed bioactive substance near the surface of produced fibers. This process depends vastly on the polarization susceptibility and solubility of incorporated substances but is the main cause of the burst release effect encountered in loaded vascular grafts obtained via direct blending technique. ${ }^{52}$

The emulsion and coaxial electrospinning are both applied to prevent burst release effect. The vesicle or core-shell structure formation causes a change in diffusion mechanism as the layers of solidified polymer acts as a diffusion barrier whereas vesicles or core becomes the bioactive substance reservoir. Changes in the elongation of the core or vesicle distribution, has a tremendous impact on the release profile as a thinner core diameter or a greater vesicle distribution causes thicker polymer-based barrier which can decrease the release rate of bioactive molecules. ${ }^{42}$ Therefore, in the case of emulsion and coaxial electrospinning, the initial stages the release profile is mostly affected by the diffusivity of bioactive substance through the polymer barrier.

Discussed burst release phase is followed by a sustained release phase with slower release rate mediated initially also by the diffusion-based mechanism. Sustained release phase is mainly a result of an increasing diffusion distance from the internal part of polymer matrix to its external regions.

The polymers used in electrospinning technique for grafting purposes are biodegradable. In long-term, the degradation process leads to subsequent surface or bulk erosion indicated by a decrease in polymer mass or fiber size which happens along a gradual regeneration of normal living tissue. ${ }^{7}$ The degradation process of the polymers used to electrospun vascular grafts is a relatively long process, therefore erosion/degradation processes are dominant release mechanisms at the last stages of graft lifespan. $^{53}$ In long-term after graft implantation, erosion-based mechanism of polymer matrix gradually decreases the diffusion distance and slowly becomes the dominant force behind the release of the bioactive substance from electrospun fibers. In the case of emulsion and coaxial electrospinning degradation of polymer chains and a gradual erosion of polymer barrier affects the passage distance and thus the diffusivity of the bioactive substance. However, when designing a long-term release medical device like bioactive substance loaded vascular graft, the impact of in vivo conditions should not be neglected. It was found that proteinase $\mathrm{K}$ and lipase PS enhance PLLA and PCL hydrolytic degradation which could increase the importance of enzymestimulated degradation in the release mechanism of bioactive substance in earlier stages of vascular graft lifespan. ${ }^{54}$

Based on presented data, both - diffusion and degradationrelated processes should be considered as potential release mechanisms of bioactive substance from electrospun grafts. In the case of long-term application of vascular grafts, the diffusion, as well as erosion/degradation, contributes to the release process. The dominant release mechanism depends on permeability, wettability and degradation profile of the polymer. The drug release from polymer graft with high permeability and characterized by a slow degradation rate is usually dominated by diffusion-driven mechanisms which produces typical bi-phase release profile. In the case when polymers with low permeability and fast degradation are applied, drug release process is more complex and is regulated by both mechanisms, resulting in a tri-phase release profile with fast release phase, diffusion-driven sustained phase and erosion-driven phase. ${ }^{55,56}$

The electrospun polymer matrix is an efficient form of drug delivery system which can provide sustained and localized release (Tables 1 and 2). Additionally, modifications of polymer matrix improve incorporation and immobilization of labile substances like growth factors or drugs for vessel regeneration.

\section{Bioactive molecules used in electrospun vascular grafts}

\subsection{Growth factors}

5.1.1 VEGF. Various growth factors are responsible for angiogenesis; however, VEGF is one the most crucial ones. From 
a number of receptors responsible for VEGF signal transduction, vascular endothelial growth factor receptors (VEGFR-1 and VEFGR-2) are the ones responsible for neo-vessel formation through angiogenesis. ${ }^{57}$ These receptors are mostly expressed on the surface of endothelial cells. ${ }^{58}$ Downstream signaling of VEGF enhances the activity of extracellular matrix degrading enzymes including proteases and collagenases which leads to the process of basal membrane degradation and neovessel sprout formation. ${ }^{59}$ Furthermore, it increases the number of epithelial progenitor cells (EPC) in general circulation, which regenerate and improve the structure as well as the function of the endothelium. ${ }^{60,61}$

Due to its remarkable importance of VEGF in angiogenesis, a study conducted by Guex et al. ${ }^{36}$ examined the possibility of VEGF immobilization on polymer scaffold for vascular tissue engineering (Table 1). In her study, Guex used a flexible linker molecule in order to increase VEGF immobilization efficiency by reducing steric hindrance. ${ }^{36}$ Cell studies on immortalized human umbilical vein endothelial cells (iHUVEC) revealed that the cells cultured nine days on VEGF immobilized scaffolds provided sustained mitogenic action. The number of cells was significantly higher for scaffold with VEGF in comparison to non-functionalized ones. Another important finding of this group demonstrated that iHUVEC attachment was increased on micro-sized fibers, in comparison to nano-sized ones which failed to enhance proliferation rate even after nine days.

A different method of active substance incorporation was selected by another group which decided to incorporate VEGF as an emulsion to PLCL fibers (Table 1). ${ }^{42}$ VEGF was dissolved in bovine serum albumin (BSA) or dextran to form a solution which then was emulsified with PLCL solution in chloroform. Both dextran ${ }^{62}$ and BSA $^{63}$ have demonstrated their stability in previous studies. Release study conducted by Tian et al. showed that dextran and BSA presence affected the release profile only during the first $24 \mathrm{~h}$. Mesenchymal stem cells (MSC) study showed no significant difference between core-shell scaffolds and pure PLCL scaffolds. However, after 10 days in both formulations of core-shell grafts, the proliferation of MSC was higher than on normal PLCL scaffolds. Growth factors could also influence the differentiation process of stem cells to other cell types. ${ }^{42}$ Despite prolonged VEGF release for 28 days, the study of Tian et al. did not demonstrate differentiation of entire MSC population to cardiomyocytes.

In another major study, Zhang et $a .^{40}$ developed doublelayered membrane composed of chitosan hydrogel/ poly(ethylene glycol)- $b$-poly(L-lactide-co-caprolactone) (PELCL) electrospun membrane loaded with VEGF as the internal layer and emulsion/PELCL electrospun membrane loaded with PDGF as the external layer (Tables 1 and 2). In this study, coaxial electrospinning was utilized to obtain double-layered scaffolds. The cumulative release of VEGF after 6 days was $66 \%$, which demonstrated that the release was more rapid than for plateletderived growth factor (PDGF), which cumulative release of $38 \%$ indicated more sustained release pattern. Two different types of cells were cultured on electrospun scaffolds and on tissueculture polystyrene (TCPS) as a control. Endothelial cells (EC)

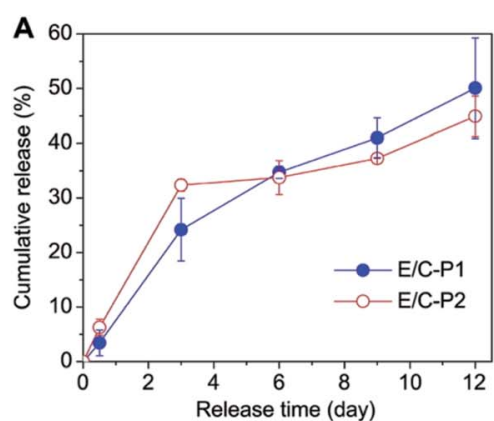

C

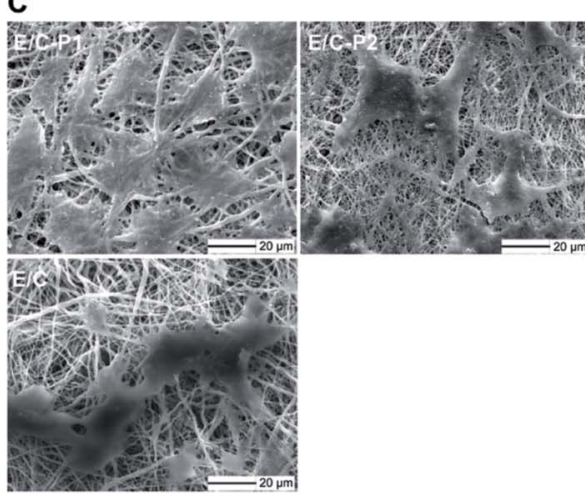

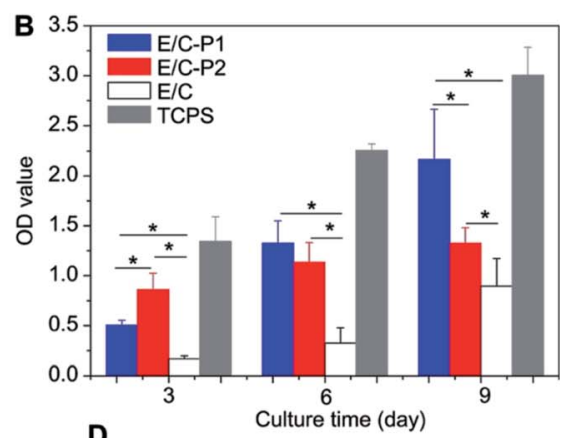

D
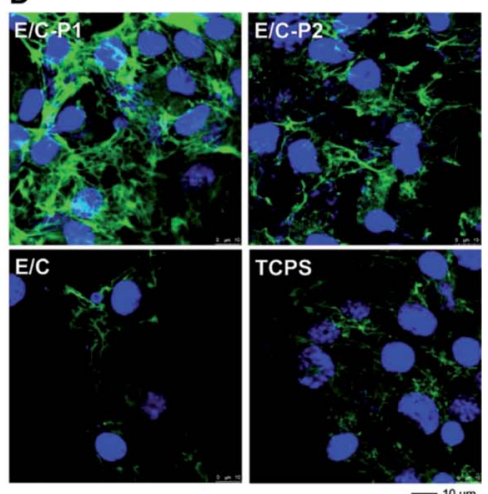

Fig. 3 The relationship between PDGF release behaviors and the VSMCs proliferation. (A) Release profiles of PDGF from the electrospun membranes; (B) CCK-8 value of VSMCs cultured on different samples $(p<0.05)$; (C) SEM micrographs for VSMCs spreading on the different electrospun membranes on the 6th day; (D) LCSM micrographs for ECM secretion of VSMCs cultured on the different electrospun membranes on day $6(1000 \AA$ A) (green, FITC labeled fibronectin; blue, DAPI stained nuclei). Reproduced from ref. 40 with permission from Elsevier. 
rapidly adhered and proliferated in the first 6 days, whereas smooth muscle cells (SMC) proliferation rate increased significantly after the $6^{\text {th }}$ day. The relationship between growth factor release from emulsion/coaxial electrospun materials $(\mathrm{E} / \mathrm{C})$, without and with different amount of heparin (P1 or P2), and vascular cells proliferation was determined (Fig. 3A-D).

In vivo studies of double layered graft implanted for 4 weeks as rabbit carotid artery replacement provided many useful findings. Neither rupture during its surgical insertion nor leakage during postoperative period was detected. The result obtained after graft explantation indicated endothelialization of luminal surface and an external layer of SMCs, however at the different percentage at different end points. Moreover, the histological analysis of cell coverage was consistent with growth factor release studies, as EC responsible for vascular homeostasis proliferated rapidly in the first week after implantation whereas SMC proliferated in the following weeks after insertion. Nevertheless, both cell types attached only to luminal end external part of the graft, which probably was the effect of insufficient porosity of the scaffold. ${ }^{40}$

A different approach was chosen by Han et al. who coelectrospun various polymers with gelatin to obtain multilayered scaffolds loaded with VEGF via direct blending and with PDGF via emulsion electrospinning (Tables 1 and 2). ${ }^{64}$ In vitro release studies demonstrated that VEGF cumulative release was higher than PDGF, which was probably caused by different loading methods and different polymers used to produce loaded fibers. This behavior was beneficial for temporal delivery of growth factors and further tissue regeneration process. Gelatin selected as a material for fiber formation and fast release of VEGF promoted adhesion and proliferation of EC on the luminal surface of electrospun scaffolds which was confirmed by increased expression of a cluster of differentiation 31 (CD31). ${ }^{65}$

5.1.2 FGF. Fibroblast Growth Factor (FGF) family is another broad group of growth factors involved in neo-vessel formation during wound healing and embryogenesis. ${ }^{66}$ They impose a pleiotropic effect on various cells including EC. This particular family of growth factors has been reported as angiogenesis stimulating factor in vascular lesions ${ }^{67}$ together with previously discussed VEGF.

Montero group investigated a new approach to fabricating aligned gelatin fibers with incorporated basic fibroblast growth factor (bFGF) (Table 1). ${ }^{68}$ The growth factor was physically immobilized within the gelatin scaffolds. The higher alignment of the fibers caused a $28 \%$ increase in an in vitro angiogenesis in comparison to scaffolds consisting of randomly oriented fibers. Moreover, greater fiber alignment was found to increase bFGF release by $11 \%$ from electrospun scaffolds with aligned fibers, which could be attributed to the swelling profiles of these fibers.

The more complex approach was applied by other researchers who managed to incorporate multiple polyplexes containing bFGF and VEGF encoding plasmids to electrospun constructs. The mixture containing growth factor encoding polyplexes were suspended in phosphate buffered saline (PBS) and emulsified in chloroform containing polyethylene oxide/ polylactic acid copolymer (PELA) and polyethylene glycol
(PEG) which were afterward electrospun. In this particular case, polyplexes of plasmid DNA (pDNA) were suspected to act as protective agents against critical process parameters. ${ }^{69}$ The release profile of obtained dual-loaded mats had three distinctive phases: an initial burst release in the first $12 \mathrm{~h}$ of the experiment, followed by a sustained release for 3 weeks and 1 week period of gradually decreasing release rate. Initial burst release was probably caused by polyplexes deposition on the surface or near to the fiber surface, which contributed to high release rate in the first hours of the release studies. After 3 weeks of a gradual dissolution of PEG, an increase in the contact surface of a polymer matrix with surrounding medium was noticed, which accelerated further polymer degradation thus enhancing the polyplexes release from the fibers.

A broader perspective of pDNA polyplexes potential was outlined by in vitro cell studies which revealed that their incorporation decreased the cell attachment to the polyplexloaded fibers. Based on these result authors concluded that the ability to properly guide the cell growth of such constructs, depend both on initial cytotoxicity of incorporated plasmid DNA and transfection rate responsible for growth factors production and thus their effect.

In another study FGF-9 was incorporated into the electrospun structures by both blend and emulsification technique. Mats obtained by both techniques showed no burst release during release study which indicated perfect entrapment of bioactive molecules within polymer fibers. Both methods of incorporation led to a sustained release of FGF-9 for 28 days. However, the cumulative release for emulsion electrospun fibers was lower compared to blend electrospun fibers and reached $37 \%$ at the end of release study. There were no significant differences in bioactivity of NIH-3T3 fibroblasts cultured on fibers prepared either by blending or emulsion techniques, while FGF-9 release profiles from both types of fibers had different characteristic. ${ }^{70}$

5.1.3 PDGF. Platelet-derived growth factor can be synthesized by a number of different cell lines and is responsible for angiogenesis. ${ }^{71}$ Together with previously mentioned FGF and VEGF this growth factor is responsible for neo-vessel maturation by enhancing the recruitment of vascular smooth muscle cells (VSMC) and pericytes. Moreover, its activity plays a crucial role in collagen production, as it stimulates fibroblasts activity. ${ }^{72}$ Additionally, to these important properties regarding angiogenesis, PDGF also induces a proinflammatory reaction in response to the vessel lining damage.

Despite being mostly used in combination with other growth factors, PDGF is also regarded as a potential candidate for a bioactive component of electrospun tissue-engineered vascular graft (TEVG). Different methods of PDGF incorporation to the electrospun graft (Tables 1 and 2) have been utilized including double-layered scaffold formation ${ }^{40}$ or immobilization on heparinized scaffold. ${ }^{73}$

In the study set out to determine PGDF effect on cell migration, ${ }^{74}$ coaxial electrospinning method was used to fabricate a PDGF-containing PLCL scaffold for sustained release of growth factor. All three structures spun with different inner phase flow rate showed burst release of PDGF-BB in the first 2 
days of the experiment, which was followed by a sustained release till the 28th day. In vitro studies conducted on VSMC revealed satisfying cell attachment when compared to normal PLCL fibers without growth factor loading. ${ }^{74}$

\subsection{Heparin}

Heparin is a well-known anticoagulant, however, only small number of trials investigated its potential in electrospun scaffolds for the cardiovascular application. Recent research has demonstrated that heparin could act as a potential linker molecule between electrospun scaffolds and various growth factors. ${ }^{51,73}$ Heparin is known to inhibit VSMC both in vitro and in vivo which can significantly reduce the severity of neointimal hyperplasia connected with grafting procedures. Over the years various mechanisms of its antiproliferative effect have been proposed however recent data showed that heparin treatment results in cGMP-dependent protein kinase activation which at least in part is responsible for VSMC proliferation inhibition. ${ }^{75}$

A research conducted by Luong-Van et al. ${ }^{37}$ produced heparin loaded PCL scaffolds by utilizing a modified direct blending technique where a non-soluble in methanol heparin was initially dissolved in water, which was then mixed with dichloromethane, before adding it to the methanol solution (Table 1). Accordingly, to other studies ${ }^{76,77}$ heparin salt addition influenced the charge deposition along the jet which enhanced its elongation during spinning process thus producing fibers with a smaller diameter. Initial, fast release rate of heparin may be beneficial in preventing the proliferation of VSMC shortly after the injury caused by endovascular operations. Proinflammatory action assay revealed that PCL fibers did not stimulate tumor necrosis factor $\alpha(\mathrm{TNF}-\alpha)$ production by the macrophages. ${ }^{37}$

Other team created heparin loaded scaffolds by using coaxial electrospinning technique (Tables 1 and 2) where the fiber core contained heparin dissolved in water whereas shell was made from poly(L-lactide-co- $\varepsilon$-caprolactone) (P(LLA-CL)). ${ }^{78}$ The release of heparin had a typical bi-phase profile with initial fast phase release followed by sustained release for 14 days. The canine model was used for long-term in vivo studies (Fig. 4A-C), due to the fact that the structure and mechanical properties of femoral artery are similar to those found in human vessels. ${ }^{78,79}$ Fig. 4A shows graft implantation procedure and graft implantation site inspection (Fig. 4B and C) by digital subtraction angiography (DSA) and color Doppler flow imaging (CDFI).

Before implantation, scaffolds underwent dynamic endothelialization. Results showed that pre-implantation endothelialization and heparin incorporation increased the patency rate about $\mathbf{1 2 . 5 \%}$ for normal scaffolds and $75 \%$ for modified scaffolds after 3 months. Non-endothelialized heparin loaded scaffolds were covered with endothelial cell only at the proximal end which was probably caused by slow cell migration rate with the blood flow.

In a recently published study, PCL and chitosan scaffold was produced by utilizing the co-electrospinning technique. ${ }^{\mathbf{8 0}}$ Heparin was physically adsorbed on the surface of produced fibers (Table 1). This research found that heparin load can be
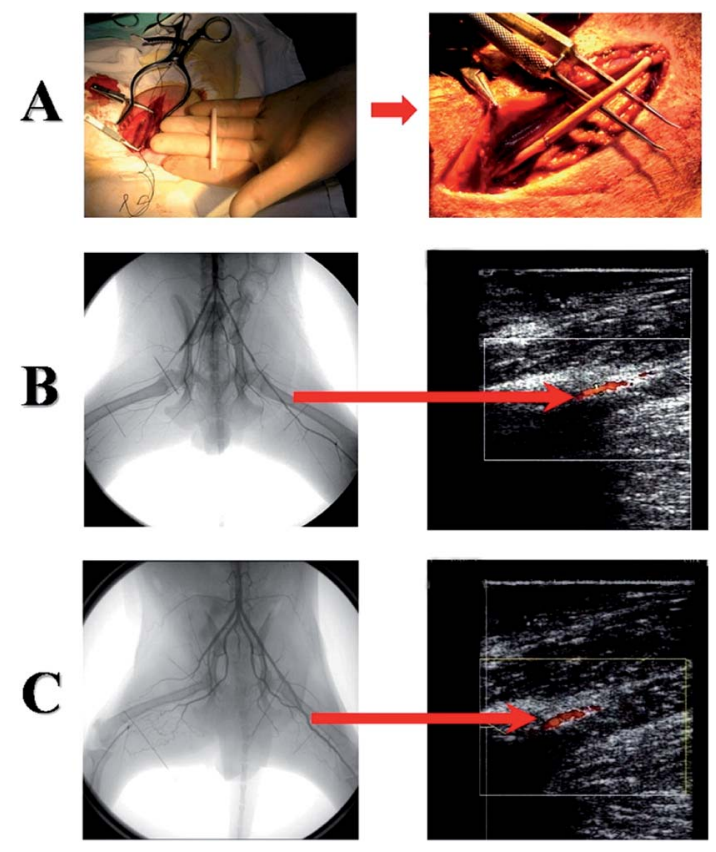

Fig. 4 (A) Surgical implantation of the electrospun tubular graft into the femoral artery of Beagle dog. Typical DSA and CDFI inspection of (B) heparin-loaded and (C) pre-endothelialized grafts after 3 months: the left femoral artery was replaced by heparin-loaded or pre-endothelialized graft, and the right one was replaced by $P(L L A-C L)$ graft. Blood flow in the left femoral artery was further traced by the CDFI image. Reprinted with permission from C. Huang, S. Wang, L. Qiu, Q. Ke, W. Zhai and X. Mo, ACS Appl. Mater. Interfaces, 2013, 5, 22202226. ${ }^{78}$ Copyright 2013 American Chemical Society.

controlled by changing the chitosan ratio within the scaffold. Moreover, due to multiple ionic bonds formed between the polymer chains of electrospun fibers, burst release was limited. The sustained release was observed for the system, from which only $26 \%$ of total heparin load was released in one month period. Heparin adsorption on the surface of the fibers extended both activated partial thromboplastin time (APTT) and thrombin time (TT) thus demonstrating high haemocompatibility. Morphological study of explanted grafts from a rat model revealed full endothelial coverage in heparin loaded scaffolds after 4 weeks. ${ }^{80}$ In contrast to normal PCL and chitosan grafts, all patent heparin loaded grafts showed clean lumen with no sign of thrombus formation.

5.2.1 Heparin as a linker molecule. Due to recent studies, heparin was also found to be a potential linker molecule between electrospun fibrous scaffolds and various growth factors. Its presence in electrospun constructs significantly increases protection of labile compounds like growth factors from various external conditions including heat, $\mathrm{pH}$, and enzymes. ${ }^{81}$ Growth factors are known to be labile compounds especially in physiological conditions, thus their immobilization via a linker molecule is considered as an option to stabilize the connection between the scaffold and multiple bioactive molecules. Protection imposed by a linker is an effect of conformational change of growth factor molecule which occurs shortly after it binds with heparin. ${ }^{\mathbf{2}}$ Moreover, such 
modification also increases the storage capacity of obtained structures and influences release pattern of bioactive substances.

Heparin use as a linker molecule was studied by Cheng. ${ }^{33}$ The study verified the platelet attachment to electrospun PLA scaffolds conjugated with heparin and showed that plasmatreated scaffolds conjugated with heparin exhibited a meaningful reduction in platelet aggregation when comparing to normal PLA scaffolds. Independently of the amount of conjugated heparin all modified scaffolds demonstrated increased bovine aorta endothelial cells (BAEC) attachment. These findings were consistent with other studies which indicated that negative charge of heparin and its water retaining capability, produce a hydrophilic environment suitable for cells. ${ }^{83}$ Since $\mathrm{NH}_{2}$ groups are considered to be potential linkers for other biomolecules such surface functionalization of currently used biodegradable polymers can improve their biocompatibility.

A similar approach was used in other research, however, no plasma treatment was involved in the heparin conjugation reaction (Table 1). ${ }^{34}$ Obtained PCL and gelatin scaffolds were cross-linked. The cross-linking process applied in this study was not only essential because of its direct impact on stability and biomechanics of electrospun polymers ${ }^{84}$ but also because it allowed more uniform distribution of heparin molecules. Furthermore, surface modification of electrospun polymer increased the loading efficiency in comparison to nonconjugated scaffolds.

The study focused on heparinization methods was continued and resulted in an immobilization of PDGF-BB into PCL/gelatin scaffold. ${ }^{85}$ The release of growth factors from heparin-conjugated structures occurred during initial phase mainly by dissociation of surface absorbed growth factor or by sustained release depending on the thermodynamic equilibrium. ${ }^{85}$ In vitro release studies showed that bioactive substance was bound to the heparinized graft by electrostatic forces, which according to the authors was the main factor contributing to a sustained dissociation of PDGF-BB from the surface of the fibers. Obtained data revealed that the release of PDGF was diameter-dependent, where fibers with small diameter released bioactive molecules at a faster rate. The probable reason for such finding was connected with a higher surface area of small diameter fibers, which was exposed to the release medium. ${ }^{86}$ Furthermore, the presence of growth factors bonded to electrospun construct was detectable even after 20 days, demonstrating the capability of heparinized scaffolds to release PDGF-BB over an extended period of time. According to this study, ${ }^{85}$ stable heparin conjugation with growth factors limited previously described antiproliferative effect of heparin on vascular smooth muscle cells. ${ }^{75,87}$

A similar method was also applied to immobilize VEGF to heparinized PCL/gelatin structures. ${ }^{51}$ Sustained release of VEGF from heparinized scaffolds was observed for 25 days, which proved high binding affinity between VEGF and heparin. The in vivo angiogenesis study in rats revealed that after 4 weeks implanted scaffold was fully covered with cells, and VEGF loading increased the vessel density in the explanted graft (Tables 1 and 2).

\subsection{NO donors}

Nitric oxide (NO) is the endothelium-derived relaxing factor responsible for vascular homeostasis. NO activity counterbalances vascular wall stiffness by inducing vasorelaxation. Additionally, to vessel contraction control, NO exerts an anti-platelet and also an anti-inflammatory effect by reducing platelet activity and leukocytes adhesion to the damaged endothelium. ${ }^{88}$ The discovery of new non-classical signaling pathways increased the complexity of NO mechanism of action. Depending on its concentration NO is capable of either promoting cell proliferation or causing an indirect cell cycle arrest or even apoptosis. Moreover, it contributes to stem cell recruitment and differentiation into desired phenotype..$^{89}$

In a study conducted by Zhang et al., coaxial electrospinning was used to obtain core-shell fibers containing NO donor - $(Z)$ $1-\{N$-butyl- $N$-[6-( $N$-butylammo-niohexyl)amino $]\}$-diazen-1-ium1,2-diolate $\left(\mathrm{DBHD} / \mathrm{N}_{2} \mathrm{O}_{2}\right){ }^{44}$ In physiological conditions this particular NO donor undergoes a spontaneous degradation with a subsequent NO release, therefore it can be regarded as a potential prodrug, where NO is an active substance responsible for clinical relevant outcomes. DBHD/ $\mathrm{N}_{2} \mathrm{O}_{2}$ itself is regarded as relatively toxic substance, thus its encapsulation within the core-shell structure of fibers was aimed to reduce its toxicity. In this study, the external layer of fibers was produced from either PCL or gelatin (Table 1). The release studies of NO from $\mathrm{DBHD} / \mathrm{N}_{2} \mathrm{O}_{2}$ incorporated to electrospun fibers revealed maximum NO flux between 2 and $5 \mathrm{~h}$. The continuous release from core-shell fibers was sustained for almost $60 \mathrm{~h}$ which was similar to non-axially spun fibers. Nevertheless, after initial burst release, core-shell structure managed to slow down the release rate in comparison to a non-coaxial structure. According to MTT assay, the cytotoxicity of $\mathrm{DBHD} / \mathrm{N}_{2} \mathrm{O}_{2}$ containing noncoaxially spun scaffolds, was apparent. Coaxial electrospinning produced core-shell structures with PCL or gelatin shell which brought more stable NO release and improved cell viability. Additionally, PCL and gelatin formed a barrier which helped to avoid direct contact of DBHD/ $\mathrm{N}_{2} \mathrm{O}_{2}$ with cells. ${ }^{44}$

In another recent study, a novel enzyme prodrug therapy (EZT) approach was adopted in electrospun vascular grafts. This approach provides means for a localized and targeted prodrug delivery ${ }^{90,91}$ In a study conducted by Wang et al. ${ }^{92}$ electrospun vascular graft was immobilized with galactosidase (Table 1), which is capable of decomposing intravenously administered NO donor with a simultaneous release of $\mathrm{NO}$ at the graft implantation site. ${ }^{93} \mathrm{NO}$ donor used in this study was galactoside NONOate (GalNONOate), which after initial decomposition conducted by a surface-immobilized enzyme, left unbounded NONOate which spontaneously decomposed to NO. ${ }^{94}$ Ex vivo catalytic properties evaluation based on an arteriovenous shunt procedure indicated that after NO prodrug administration, fluorescent dye-labeled grafts demonstrated evident green fluorescence in contrast to the control groups, indicating localized release of NO. Electrospun grafts were implanted as abdominal artery substitutes in rats (Fig. 5A-G), where platelet adhesion (Fig. 5D1, D2, F1 and F2) and graft endothelialization (Fig. 5E1 and E2) was observed. Quantitative analysis revealed that thickness of neotissue and coverage 

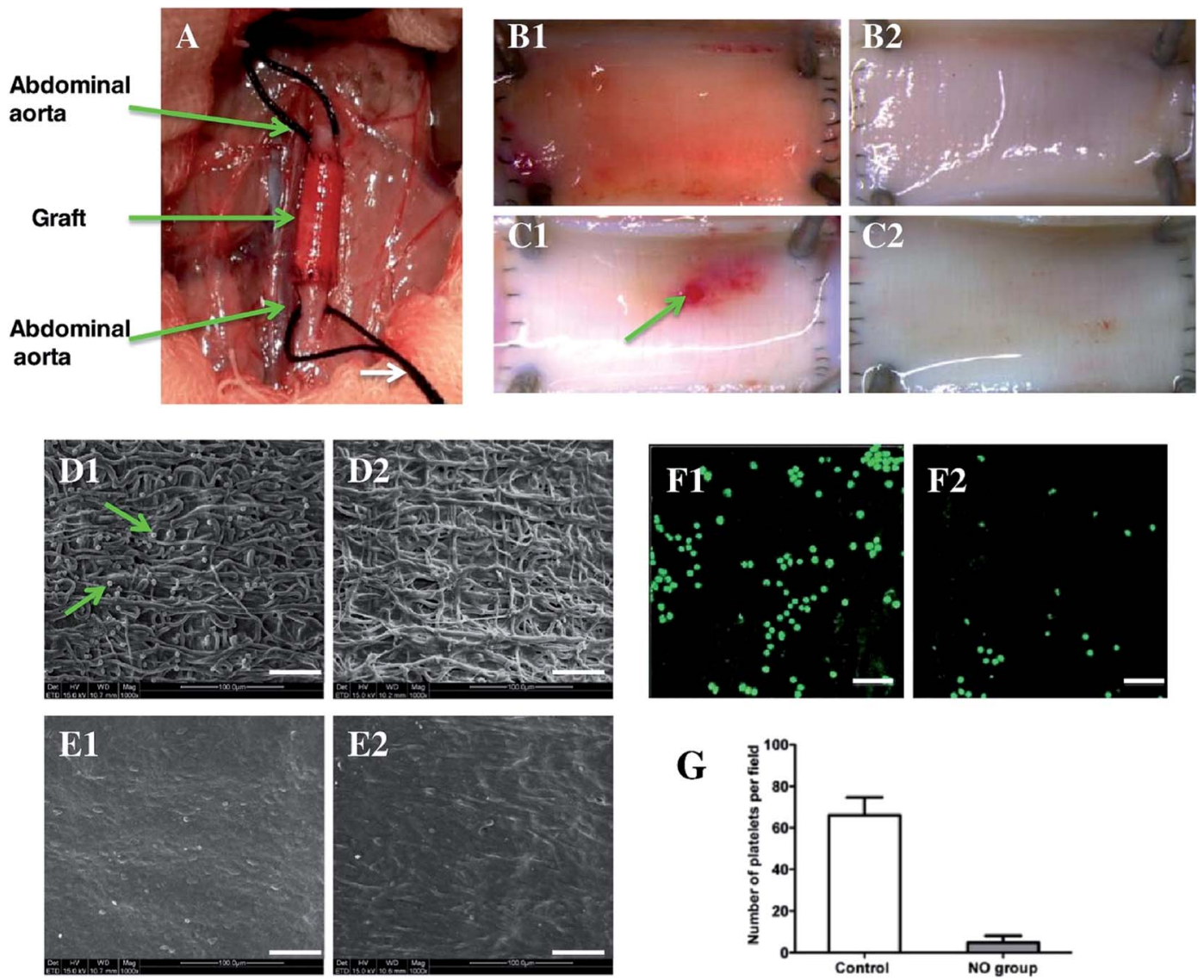

Fig. 5 Implantation of enzyme-functionalized vascular grafts by replacing a part of the rat abdominal aorta (A), and the luminal surface of explanted vascular grafts at day 7 (B1, B2) and day 14 (C1, C2) without (B1, C1) and with (B2, C2) administrated NO-prodrug observed by a stereomicroscope. SEM images show the platelet adhesion and endothelialization of the enzyme-functionalized PCL grafts without (D1, E1) and with NO-prodrug administration (D2, E2) over days 7 (D1, D2) and 14 (E1, E2). CLSM images show the mepacrine-stained platelets adhered on explanted grafts over day 7 without (F1) and with NO-prodrug administration (F2), and the correlative statistical analysis (G). Scale bar is $50 \mu \mathrm{m}$. Data are expressed as mean \pm SEM. Reproduced from ref. 92 with permission from Elsevier.

ratio was significantly higher in the experimental group (with GalNONOate administration) than in control (without Gal-NONOate administration) after 2 weeks after implantation. However, after one month no significant difference was detected. ${ }^{92}$

A study conducted by Andukuri et al. produced a hybrid scaffold consisting of electrospun PCL and peptide amphiphiles (PAs). ${ }^{95}$ In the discussed study two different PA were synthesized. Each of them contained either a laminin-derived Tyr-IleGly-Ser-Arg endothelial cell adhesive ligands (YIGSR) or polylysine sequence (KKKKK) acting as a nitric oxide (NO) donor. After mixing PAs were reacted with pure NO and then selfassembled on the electrospun PCL scaffold to generate a hybrid scaffold (Table 1). Initial burst release occurred during the first $48 \mathrm{~h}$ and was followed by 4 weeks of sustained release. Cell studies conducted on HUVEC and aorta smooth muscle cells (AoSMC) showed that hybrid scaffolds with NO donor enhanced endothelial cells adhesion to scaffold fibers, while simultaneously they did not support smooth muscle cell adhesion. These findings are consistent with other research on the NO control over vascular cells homeostasis. ${ }^{96}$ Moreover, NOreleasing hybrid scaffolds were found to decrease platelet adhesion, thus limiting the potential thrombosis.

\subsection{Active pharmaceutical ingredients}

5.4.1 Antiplatelet and antithrombotic agents. Acetylsalicylic acid (ASA) is a cornerstone of a dual antiplatelet therapy used as prevention in patients with atherosclerotic vascular disease. ${ }^{97}$ By influencing platelet enzyme - cyclooxygenase 1 (COX-1), which catalyzes the conversion of arachidonic acid to prostaglandin H2, ASA affects the platelet aggregation. Del Gaudio et al. were first who managed to incorporate ASA to electrospun tubular scaffolds via direct blending. ${ }^{98}$ According to the tensile strength measurements, ASA loading did not influence the mechanical properties of electrospun tubular structures (Table 1). Sustained release of ASA from tubular scaffolds was obtained for a one-week period. Short-term analysis of antiplatelet effect revealed that $1 \%$ ASA incorporation decreased platelet adhesion to the electrospun fibers. However, this effect was mitigated in higher concentration (5\% ASA) which according to the authors was a result of less favorable morphological properties and non-homogenous distribution of ASA within the fibers. Only results obtained after $6 \mathrm{~h}$ for $10 \%$ ASA concentration were statistically significant and showed observable antiplatelet effect. 

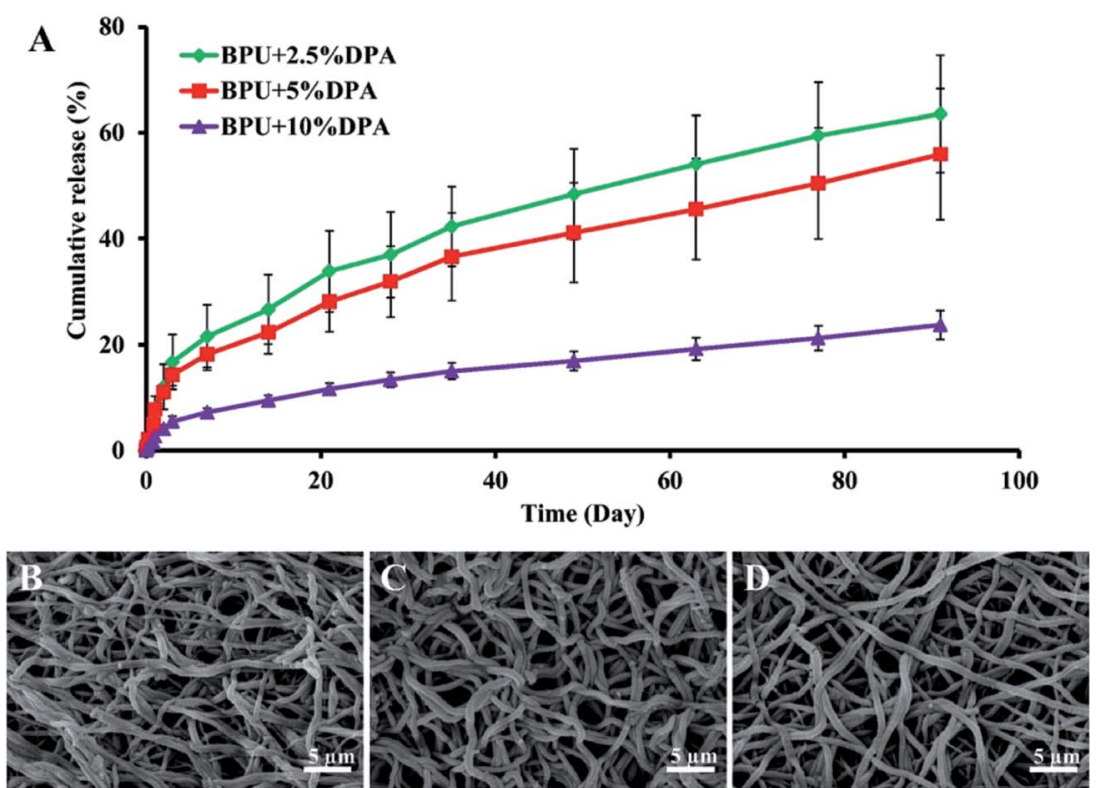

Fig. 6 (A) In vitro DPA release curves of DPA-loaded BPU scaffolds up to 91 days in PBS at $37^{\circ} \mathrm{C}$. SEM images exhibited fiber morphologies of (B) $\mathrm{BPU}+2.5 \%$ DPA, (C) BPU + 5\% DPA and (D) BPU + 10\% DPA after 91 day release. Reproduced from ref. 102 with permission from Elsevier.

Dipyridamole (DPA) is an antithrombotic drug which is able to reduce platelet aggregation and over-proliferation of SMC by inhibiting adenosine uptake via phosphodiesterase activation inhibition in these cells, resulting in the intracellular increase of cyclic adenosine monophosphate (cAMP) and cGMP levels. ${ }^{99}$ The same mechanism was reported to protect endothelial cells and stimulate their growth. ${ }^{99,100}$ Based on these pleiotropic effects of DPA it was hypothesized that its incorporation may improve the clinical outcome of electrospun vascular grafts. ${ }^{101}$
Direct blend technique was used to incorporate DPA into biodegradable polyurethane urea (BPU) electrospun scaffolds (Table 1). ${ }^{102}$ Obtained structures showed improved mechanical properties of DPA incorporated scaffolds which according to the authors was a result of hydrogen bond formation between hydroxyl groups of DPA and urea groups of BPU. The burst release was limited, followed by a sustained release of DPA for 91 days with only few fiber defects found by SEM micrographs analysis (Fig. 6A-D).


E
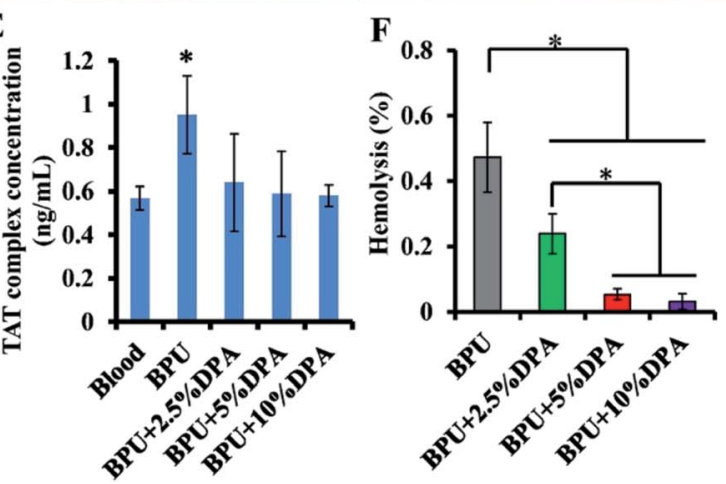

Fig. 7 Digital images to show human blood clot formation with time of (A) human blood, (B) BPU alone in the human blood and (C) BPU $+10 \%$ DPA in the human blood. (D) The absorbance at $540 \mathrm{~nm}$ of the lysate of human blood contacted with the scaffold. (E) The TAT complex concentration in PPP after the scaffold has been in contact with human blood. The control is human blood alone. (F) Human blood hemolysis percentages of the scaffolds. Reproduced from ref. 102 with permission from Elsevier. 
Limited burst release effect and long sustained release phase were probably caused by a proper incorporation of hydrophobic DPA which matched hydrophobic moieties of BPU and placed the active substance within the fiber structure. Both $5 \%$ and $10 \%$ DPA concentration caused SMC proliferation inhibition in a dose-dependent manner which was consistent with another study. ${ }^{103}$ Moreover, EC proliferation was improved in comparison to normal BPU scaffolds, but exact concentration needed to obtain this effect is still an area of discussion.

Human blood contact assessment was also conducted (Fig. 7A-F). Compared to the BPU alone, the DPA-loaded BPU scaffolds had improved non-thrombogenicity. This study did not show dose dependency of DPA antithrombotic effect, therefore antiproliferative and antithrombogenic effects probably occurred at different dosage of DPA. ${ }^{102}$

5.4.2 Antimicrotubule agent. Paclitaxel (PTX) action prevents microtubule formation by binding to B subunit of tubulin and thus disrupts cell mitosis at the G2/M stage, which further disrupts basic cell functions. ${ }^{\mathbf{1 0 4}}$ Therefore, PTX is mostly used in chemotherapy in order to impose antiproliferative effect to affect and combat over-proliferating cancer cells. Based on this specific mechanism of action PTX was incorporated to vascular stents in order to limit neointimal hyperplasia formation. ${ }^{105}$ In the study conducted by Innocente et al. direct blending technique was used to incorporate different amounts of PTX to PCL scaffolds (Tables 1 and 2). ${ }^{\mathbf{1 0 6}}$ The burst release was limited and followed by 22 days of sustained release when $60 \%$ of PTX was eluted from the graft. In vivo studies were conducted in a rat model. Analyzed blood samples demonstrated that systematic concentration of PTX was below the detection limit, therefore should not cause general side effects. In vivo study showed a delay in the endothelial coverage as well as the cellular ingrowth till the $12^{\text {th }}$ week of the experiment, probably caused by PTX activity. Still, a significant decrease of neointimal hyperplasia was found in explanted grafts at each time point of the in vivo study. Furthermore, these observations together with the previous study revealed the potential advantage of PTX loaded, electrospun graft over commercially available expanded polytetrafluoroethylene (ePTFE) grafts. ${ }^{107}$

5.4.3 Statins. Statins are commercially available 3-hydroxy3-methylglutaryl-coenzyme A (HMG-CoA) reductase inhibitors used in hypercholesterolemia. Additionally, to their most widely known effects on cholesterol levels, they are also capable of promoting endothelial function and thus reducing platelet activation with concurrent inhibition of SMC proliferation. ${ }^{\mathbf{1 0 8 , 1 0 9}}$ Moreover, accumulated evidence suggests that statins affect EPC mobilization and proliferation, independently of their main mechanism of action. ${ }^{\mathbf{1 1 0}}$ By influencing apolipoprotein-III statins also impose an anti-inflammatory effect by blocking monocyte adhesion to the endothelial cells. ${ }^{\mathbf{1 1 1}}$

Lee et al. used electrospun PLGA tubes containing rosuvastatin as a drug delivering coating for bare metal stents (Tables 1 and 2). ${ }^{112}$ After electrospinning obtained fibrous tube was mounted on the outside of a commercially available bare metal stent. In vitro platelet adhesion to obtained fibers were found to be significantly lower for fiber with 2 and $5 \mu \mathrm{g} \mathrm{mm}{ }^{-2}$ rosuvastatin than for normal PLGA fibers and the antiplatelet effect was directly proportional to the concentration of the drug. The in vitro drug release studies showed two-phase release profile. The stable and sustained release of rosuvastatin were observed until the $4^{\text {th }}$ week of the experiment. Hybrid stent covered with rosuvastatin containing coating was implanted in the rabbit descending abdominal aorta. In vivo study of endothelial coverage showed that re-endothelialization was significantly greater in 2 and $5 \mu \mathrm{g} \mathrm{mm}{ }^{-2}$ hybrid stents $(95.5 \pm 0.4 \%$ and 99.1 $\pm 0.3 \%$ respectively) than in rosuvastatin-free hybrid stent ( 86.0 $\pm 0.7 \%$ ). After four weeks, all three groups exhibited significantly different vascular inflammatory responses, where rosuvastatin loaded hybrid stents showed two times lower response than the control one. Such result could be the effect of either drug-induced improved endothelial function or reduced platelet activation. ${ }^{\mathbf{1 1 3 , 1 1 4}}$

Similarly, another representative of (HMG-CoA) reductase inhibitors - simvastatin was directly blended and electrospun with polyethylene oxide (PEO). However, the obtained fibers with the highest investigated 1:4 drug-carrier ratio showed a sustained release over a period of 12 h. ${ }^{115}$

5.4.4 Anti-inflammatory agents. Dexamethasone (Dex) is an anti-inflammatory glucocorticoid drug which lacks sodiumretaining property and makes it suitable for patients experiencing cardiovascular problems. ${ }^{\mathbf{1 1 6}}$ Dex-loaded silk fibroin (SF)/ PEO scaffolds were formed by applying emulsion electrospinning, where Dex was dissolved in plant oil and then emulsified with a water solution of SF/PEO. ${ }^{\mathbf{1 1 7}}$ The presented study investigated the possibility of green electrospinning without the use of organic solvents (Table 1). Water evaporation rate was improved by manipulating the ambient conditions by setting the temperature to $37{ }^{\circ} \mathrm{C}$ and maintaining the humidity between 20-30\%. According to in vitro release studies both materials, obtained by direct blending and emulsion technique showed burst release for the first hours of experiment, followed by more sustained release for $192 \mathrm{~h}$. Due to core-shell structure formation with a hydrophobic core containing Dex, the release rate in burst phase, as well as the total release, was lower for scaffolds obtained by emulsion electrospinning. In vitro studies combined with SEM analysis revealed that Dex delivery inhibited the lipopolysaccharide (LPS)-induced damage to endothelial cells whereas the normal SF/PEO scaffolds failed to limit the cell damage caused by LPS.

5.4.5 Antibiotics. As the systematic treatment often fails to address bacterial infection after vascular intervention antibiotic-eluting electrospun graft were developed. Vancomycin is a potent glycopeptide antibiotic, which inhibits bacterial cell-wall synthesis, effective against Gram positive bacteria. It is used for complicated infections caused by bacteria suspected to be resistant to other antibiotics. Moreover, vancomycin off-label use includes preoperative and surgical antimicrobial prophylaxis. Antibiotic-loaded fibers were prepared by electrospinning vancomycin and PLGA in hexafluoroisopropanol (HFIP) on a commercially available vascular graft (Tables 1 and 2). ${ }^{\mathbf{1 1 8}}$ Vancomycin loaded fibers provided a sustainable triphasic release for more than 30 days in vitro. Nonetheless, an initial burst release of vancomycin could be a favorable feature, as it is important for effective bacteria eradication. ${ }^{119}$ In vivo studies 
conducted in a rabbit model also indicated similar release time period and showed significantly higher local tissue concentration of active substance than its concentrations in and at the graft, which indicated limited systematic effect.

\section{Perspectives}

As can be clearly seen from presented evidence in the past 10 years the scientific community has witnessed an outstanding progress in the field of electrospinning and tissue engineering. However, despite a significant number of different electrospun delivery system formulations successfully implanted to animal models, still, no marketed products are available. There are some exceptions, which utilize non-loaded electrospinning materials like Papyrus - a coronary balloon-expandable stent system marketed by Biotronik ${ }^{120}$ and $\mathrm{AVflo}^{\mathrm{TM}}$ a vascular access graft produced by Nicast, ${ }^{121}$ both with CE mark. Also, Zeus Bioweb ${ }^{\circledR}$ technology is an intriguing concept with a number of potential medical applications. Recently, Biostage electrospun product - Cellspan ${ }^{\mathrm{TM}}$ esophageal implant was granted Orphan Drug Designation by the U.S. Food and Drug Administration (FDA) and the advancement into phase 1 human clinical studies. Additionally, at the end of January 2017, FDA decision allowed Xeltis after successful animal study to initiate the Xplore-II clinical trial for its electrospun pulmonary valve in the USA. ${ }^{122}$ The fibrous structure of this device will enable cardiovascular tissue restoration once implanted.

Still, human clinical trials of electrospun vascular grafts are scarce. Nevertheless, some of the current research might help to address some of the issues responsible for such state of matter.

Cell infiltration and proliferation within the vascular graft, just like in physiological angiogenesis are guided by the timedependent action of different growth factors. However, excessive revascularization may contribute to neointimal hyperplasia which needs to be counterbalanced by suppressing growth factors activity. Sequential delivery of growth factors has been shown to be responsible for proper vascularization process.

Therefore one of the greatest challenges for future vascular grafts is the spatiotemporal delivery of bioactive substances in response to natural signaling triggers. ${ }^{123}$ This issue is already an area of extensive research thanks to the introduction of stimuliresponsive polymers. Despite being a novelty such polymers are already extensively studied as they may enable a more controllable release of the bioactive substance. Various external triggers may be used to activate to generate a change in polymer structure with subsequent release of the incorporated substance.

As the $\mathrm{pH}$ of arterial blood pressure is in a very narrow range between 7.38 and 7.42, pH-responsive fibers can detect and respond to any alterations in $\mathrm{pH}$ homeostasis. Polymers containing bonds susceptible to $\mathrm{pH}$ fluctuations show faster degradation rate under acidic conditions which facilitate hydrolysis of acetal of ester groups. ${ }^{124}$ As a result, this degradation-based mechanism increases the elution rate of the bioactive substance from polymer scaffold. In general, in the case of the cardiovascular system, lowered blood $\mathrm{pH}$ indicates either inflammation or more severe conditions including myocardial infarction. Therefore, $\mathrm{pH}$ could act as a potential trigger for a controllable release of bioactive substances from the implanted graft. ${ }^{125,126}$ Additionally, it could enable spatiotemporal delivery of angiogenesis promoting factors like bFGF. ${ }^{127}$

An interesting approach for bioactive substance delivery involves thermo-responsive particles incorporation into electrospun fibers, which after periodic variation in temperature would release the bioactive substances in a controllable manner. ${ }^{128}$ These smart scaffolds with predefined temperatureinduced phase transition may be an interesting delivery technique and a new approach for a localized delivery of proteins. ${ }^{129,130}$

Currently, external triggers also gain attention and are often applied to electrospun materials design, as they are explored as a potential improvement in these delivery systems. ${ }^{131}$

An electric current was identified as a potential trigger for swelling and shrinking of polymer based constructs. By increasing the applied voltage, more carboxylic acid groups of polymers becomes ionized which leads to greater repulsion between fibers and results in fiber swelling. ${ }^{124,132}$ Because of these properties, modified electro-responsive fibers are utilized to control thrombogenicity and inflammatory responses of electrospun vascular grafts. The heparinized PCL fibers loaded with polypyrrole showed a distinct response to the low alternating current which led to significant decrease in platelet activation and leukocyte adhesion. ${ }^{133}$

Other potential methods for improving vascular graft patency are connected with gene and plasmid delivery. Electrospun scaffolds modified by simple physical adsorption of small interfering RNA (siRNA) could target genes associated with neointimal hyperplasia. ${ }^{134}$ Gene silencing via thrombospondin-2 (TSP-2) siRNA delivery in electrospun polyethylene terephthalate (PET) graft was found to decrease TSP-2 gene expression, which contributes to anastomosis of implanted biomaterials. ${ }^{135}$ Electrospraying technique is an emerging method for nano and microparticles preparation. ${ }^{136}$ It has been verified as an efficient method for drug loading to microparticles. However, it becomes apparent that it is also suitable for plasmid DNA loading to microparticles. ${ }^{137}$ Electrospinning technique coupled with electrospraying provides new ways of plasmid-containing microparticles incorporation to vascular grafts, which can induce rapid endothelialization of vascular grafts. ${ }^{138}$ In order to mimic the complex signaling pathway guiding proper neovessel formation development of novel manufacturing techniques of multiple active substance delivery systems should be one of the priorities of future research. Therefore nano and micro sized particles are widely investigated as new controlled release formulations for delivering single or multiple active substances in a sustainable manner. ${ }^{139,140}$

New active substance selection ${ }^{140}$ targeting vascular reconstruction issues is also an interesting approach to developing new drug delivering vascular grafts. In one recent study, ferulic acid (Angelica sp.) and astragaloside IV (Astragalus sp.) were loaded to electrospun scaffolds to promote revascularization. The addition of these plant-derived active substances increased 
cell viability of EC and VSMC in vitro and promoted angiogenesis after subcutaneous implantation with mild inflammatory reaction. ${ }^{\mathbf{1 4 1}}$ Additionally, processing of the new candidates for bioactive substance incorporation to electrospun materials could improve the basic knowledge of process parameters which is essential for further developments in this field.

Furthermore, several issues regarding electrospinning process still need to be addressed to fully exploit the potential of these scaffold-based delivery systems. Proper process parameters optimization is needed in order to improve reproducibility and clinical performance of electrospun scaffolds before starting the first clinical study.

To date most of the conducted release studies of substances incorporated to vascular grafts were performed in vitro, therefore to obtain more preclinical data, a greater number of in vivo studies need to be performed. The endpoint of in vivo studies should also consider the long-term use of vascular grafts which should lead to full tissue regeneration.

Additionally, it is of extreme importance to manage to scale up the whole process to the industrial level. Careful selection of used materials and electrospinning parameters need full optimization to obtain method fully transmissible to large scale production. Therefore, drug eluting vascular grafts design should also involve requirements associated with viability maintenance during storage period prior to final use of electrospun grafts.

Studies in mentioned areas are crucial for developing first clinically available and successful electrospun vascular graft.

\section{Conclusion}

In the next years, cardiovascular events will contribute to an even higher number of deaths than they cause nowadays. Concurrently with these figures and continuous rise in the percentage of elderly patients in developed countries population, the demand for novel vascular grafts will increase, as presently available non-degradable and degradable prosthesis may not reach all of the requirements of modern blood vessel replacement surgeries. The development of better biodegradable substitutes of presently available vascular grafts requires more complex approach. Additionally, novel grafts design should incorporate new concepts enabling cell infiltration and improving control over the process of neovessel formation. As has been shown in this paper the well-known advantages of electrospun scaffolds with their high porosity and interconnected structure might be complemented by utilizing their high surface area to volume ratio as a basis for active substance reservoir.

Moreover, known issues connected with grafting procedures like the excessive growth of neointima, insufficient cell penetration and proliferation leading to vascular graft failure might be easily addressed by proper active substance incorporation to the electrospun grafts. Nowadays, it became even more apparent that this strategy combining scaffold and delivery system is best-suited for tissue engineered purposes.

This paper summarizes new ways of surface functionalization and active substance incorporation to electrospun

scaffolds. A rich variety of utilized polymers, emulsion, and coaxial electrospinning, as well as other setup modifications, allow more efficient application of labile compounds like proteins and growth factors. Furthermore, all these elements allow to obtain more controllable graft structure, providing means for a sustained release of bioactive substance which is well-suited for long-term application of vascular grafts. Due to its versatile nature, electrospinning technique is starting to become a new tool for novel drug delivery systems production for future vascular grafts.

\section{Abbreviations}

AoSMC
APTT
ASA
BAEC
BPU
BSA
cAMP
CCK- 8
CD31
CDFI
cGMP
COX
COX
DBHD $/ \mathrm{N}_{2} \mathrm{O}$

DPA

EC

DSA

ECM

EPC

EZT

FGF

bFGF

pbFGF

Gal-NONOate

HMG-CoA

HFIP

hSMC

HUVEC

iHUVEC

pHUVEC

\section{KKKKK}

LCSM

LPS

MSC

NIH-3T3

NO

PBS

PAs

PCL

PDNA

PEG

PELA
Aorta smooth muscle cells

Activated partial thromboplastin time

Acetylsalicylic acid

Bovine aorta endothelial cells

Biodegradable polyurethane urea

Bovine serum albumin

Cyclic adenosine monophosphate

Cell counting kit-8 assay

Cluster of differentiation 31

Color Doppler flow imaging

Cyclic guanosine monophosphate

Cyclooxygenase

Cyclooxygenase

(Z)-1-\{N-butyl- $N$-[6-( $N$-butylammoniohexyl)

amino]\}-diazen-1-ium-1,2-diolate

Dipyridamole

Endothelial cells

Digital subtraction angiography

Extracellular matrix

Endothelial progenitor cells

Enzymes prodrug therapy

Fibroblast growth factor

Basic fibroblast growth factor

Plasmid basic fibroblast growth factor

Galactoside NONOate

3-Hydroxy-3-methylglutaryl-coenzyme A

Hexafluoroisopropanol

Human smooth muscle cells

Human umbilical vein endothelial cells

Immortalized human umbilical vein

endothelial cells

Primary human umbilical vein endothelial cells

Polylysine sequence

Laser confocal scanning microscopy

Lipopolysaccharide

Mesenchymal stem cells

Mouse embryonic fibroblast cells

Nitric oxide

Phosphate buffered saline

Peptide amphiphiles

Polycaprolactone

Plasmid DNA

Polyethylene glycol

Polyethylene oxide/polylactic acid copolymer 
PELCL

Poly(ethylene glycol)- $b$-poly(L-lactide-cocaprolactone)

PET Polyethylene terephthalate

PDGF Platelet-derived growth factor

PDGF-BB Platelet-derived growth factor BB homodimer

PEO Polyethylene oxide

PIEC Porcine hip artery endothelial cells

PLCL Poly(lacticacid-co-caprolactone)

P(LLA-CL) Poly(L-lactide-co- $\varepsilon$-caprolactone)

PLA

PLGA

PPP

ePTFE

PTX

SF

SMC

siRNA

TAT

TCPS

TEVG

TNF- $\alpha$

TSP-2

TT

VEGF

pVEGF

VEGFR

VSMC

YIGSR

Poly(lactic acid)

Poly(lactic-co-glycolic acid), poly(glycolic acid)

Platelet poor plasma

Expanded polytetrafluoroethylene

Paclitaxel

Silk fibroin

Smooth muscle cells

Small interfering RNA

Thrombin-antithrombin complex

Tissue-culture polystyrene

Tissue engineered vascular graft

Tumor necrosis factor $\alpha$

Thrombospondin-2

Thrombin time

Vascular endothelial growth factor

Plasmid vascular endothelial growth factor

Vascular endothelial growth factor receptor

Vascular smooth muscle cells

Laminin-derived Tyr-Ile-Gly-Ser-Arg endothelial cell adhesive ligands

\section{Acknowledgements}

This research was supported by the National Science Centre grants (PRELUDIUM9: UMO-2015/17/N/ST8/00102).

\section{References}

1 M. Writing Group Members, D. Mozaffarian, E. J. Benjamin, A. S. Go, D. K. Arnett, M. J. Blaha, M. Cushman, S. R. Das, S. de Ferranti, J.-P. Després, H. J. Fullerton, V. J. Howard, M. D. Huffman, C. R. Isasi, M. C. Jiménez, S. E. Judd, B. M. Kissela, J. H. Lichtman, L. D. Lisabeth, S. Liu, R. H. Mackey, D. J. Magid, D. K. McGuire, E. R. Mohler, C. S. Moy, P. Muntner, M. E. Mussolino, K. Nasir, R. W. Neumar, G. Nichol, L. Palaniappan, D. K. Pandey, M. J. Reeves, C. J. Rodriguez, W. Rosamond, P. D. Sorlie, J. Stein, A. Towfighi, T. N. Turan, S. S. Virani, D. Woo, R. W. Yeh, M. B. Turner, American Heart Association Statistics Committee and Stroke Statistics Subcommittee, Circulation, 2016, 133, 447-454.

2 C. V. C. Bouten, P. Y. W. Dankers, A. Driessen-Mol, S. Pedron, A. M. A. Brizard and F. P. T. Baaijens, Adv. Drug Delivery Rev., 2011, 63, 221-241.

3 S. G. Wise, M. J. Byrom, A. Waterhouse, P. G. Bannon, A. S. Weiss and M. K. C. Ng, Acta Biomater., 2011, 7, 295-303.

4 K. A. McKenna, M. T. Hinds, R. C. Sarao, P.-C. Wu, C. L. Maslen, R. W. Glanville, D. Babcock and K. W. Gregory, Acta Biomater., 2012, 8, 225-233.
5 P. Klinkert, P. N. Post, P. J. Breslau and J. H. van Bockel, Eur. J. Vasc. Endovasc. Surg., 2004, 27, 357-362.

6 E. Chung, L. M. Ricles, R. S. Stowers, S. Y. Nam, S. Y. Emelianov and L. J. Suggs, Nano Today, 2012, 7, 514531.

7 A. Hasan, A. Memic, N. Annabi, M. Hossain, A. Paul, M. R. Dokmeci, F. Dehghani and A. Khademhosseini, Acta Biomater., 2014, 10, 11-25.

8 K. A. Rocco, M. W. Maxfield, C. A. Best, E. W. Dean and C. K. Breuer, Tissue Eng., Part B, 2014, 20, 628-640.

9 N. L'Heureux, S. Pâquet, R. Labbé, L. Germain and F. A. Auger, FASEB J., 1998, 12, 47-56.

10 N. L'Heureux, N. Dusserre, G. Konig, B. Victor, P. Keire, T. N. Wight, N. A. F. Chronos, A. E. Kyles, C. R. Gregory, G. Hoyt, R. C. Robbins and T. N. McAllister, Nat. Med., 2006, 12, 361-365.

11 T. Shin'oka, Y. Imai and Y. Ikada, N. Engl. J. Med., 2001, 344, 532-533.

12 M. A. Cleary, E. Geiger, C. Grady, C. Best, Y. Naito and C. Breuer, Trends Mol. Med., 2012, 18, 394-404.

13 V. S. LeBleu, B. Macdonald and R. Kalluri, Exp. Biol. Med., 2007, 232, 1121-1129.

14 S. Brody, T. Anilkumar, S. Liliensiek, J. A. Last, C. J. Murphy and A. Pandit, Tissue Eng., 2006, 12, 413-421.

15 C. Spadaccio, M. Chello, M. Trombetta, A. Rainer, Y. Toyoda and J. A. Genovese, J. Cell. Mol. Med., 2009, 13, 422-439.

16 E. A. Phelps and A. J. Garcia, Regener. Med., 2009, 4, 65-80.

17 D. Kai, S. S. Liow and X. J. Loh, Mater. Sci. Eng., C, 2014, 45, 659-670.

18 S. Heydarkhan-Hagvall, K. Schenke-Layland, A. P. Dhanasopon, F. Rofail, H. Smith, B. M. Wu, R. Shemin, R. E. Beygui and W. R. MacLellan, Biomaterials, 2008, 29, 2907-2914.

19 N. G. Rim, C. S. Shin and H. Shin, Biomed. Mater., 2013, 8, 14102.

20 S. Zhong, W. E. Teo, X. Zhu, R. W. Beuerman, S. Ramakrishna and L. Y. L. Yung, J. Biomed. Mater. Res., Part A, 2006, 79, 456-463.

21 B. Sun, Y. Z. Long, H. D. Zhang, M. M. Li, J. L. Duvail, X. Y. Jiang and H. L. Yin, Prog. Polym. Sci., 2014, 39, 862890.

22 Z.-M. Huang, Y.-Z. Zhang, M. Kotaki and S. Ramakrishna, Compos. Sci. Technol., 2003, 63, 2223-2253.

23 C. of Europe, European Pharmacopoeia 8th Edition, Council of Europe, Strasbourg, 2013.

24 C. Errico, N. Detta, D. Puppi, A. M. Piras, F. Chiellini and E. Chiellini, Polym. Int., 2011, 60, 1162-1166.

25 A. Baji, Y.-W. Mai, S.-C. Wong, M. Abtahi and P. Chen, Compos. Sci. Technol., 2010, 70, 703-718.

26 C. P. Barnes, S. A. Sell, E. D. Boland, D. G. Simpson and G. L. Bowlin, Adv. Drug Delivery Rev., 2007, 59, 1413-1433.

27 K. Kim, Y. K. Luu, C. Chang, D. Fang, B. S. Hsiao, B. Chu and M. Hadjiargyrou, J. Controlled Release, 2004, 98, 47-56.

28 P. Zahedi, Z. Karami, I. Rezaeian, S.-H. Jafari, P. Mahdaviani, A. H. Abdolghaffari and M. Abdollahi, J. Appl. Polym. Sci., 2012, 124, 4174-4183. 
29 X. Xu, X. Chen, P. Ma, X. Wang and X. Jing, Eur. J. Pharm. Biopharm., 2008, 70, 165-170.

30 J. Xie, R. S. Tan and C.-H. Wang, J. Biomed. Mater. Res., Part A, 2008, 85, 897-908.

31 T. Lu, X. Jing, X. Song and X. Wang, J. Appl. Polym. Sci., 2012, 123, 209-217.

32 X. Dai, J. Du Plessis, L. Kyratzis, G. Maurdev, M. Huson and C. Coombs, Plasma Processes Polym., 2009, 6, 490-497.

33 Q. Cheng, K. Komvopoulos and S. Li, J. Biomed. Mater. Res., Part A, 2014, 102, 1408-1414.

34 J. Lee, J. J. Yoo, A. Atala and S. J. Lee, Acta Biomater., 2012, 8, 2549-2558.

35 W. Ji, Y. Sun, F. Yang, J. J. J. P. van den Beucken, M. Fan, Z. Chen and J. A. Jansen, Pharm. Res., 2011, 28, 1259-1272.

36 A. G. Guex, D. Hegemann, M. N. Giraud, H. T. Tevaearai, A. M. Popa, R. M. Rossi and G. Fortunato, Colloids Surf., $B, 2014,123,724-733$.

37 E. Luong-Van, L. Grøndahl, K. N. Chua, K. W. Leong, V. Nurcombe and S. M. Cool, Biomaterials, 2006, 27, 2042-2050.

38 R. Dave, P. Jayaraj, P. K. Ajikumar, H. Joshi, T. Mathews and V. P. Venugopalan, J. Biomater. Sci., Polym. Ed., 2013, 24, 1305-1319.

39 X. Xu, X. Zhuang, X. Chen, X. Wang, L. Yang and X. Jing, Macromol. Rapid Commun., 2006, 27, 1637-1642.

40 H. Zhang, X. Jia, F. Han, J. Zhao, Y. Zhao, Y. Fan and X. Yuan, Biomaterials, 2013, 34, 2202-2212.

41 S. He, T. Xia, H. Wang, L. Wei, X. Luo and X. Li, Acta Biomater., 2012, 8, 2659-2669.

42 L. Tian, M. P. Prabhakaran, X. Ding, D. Kai and S. Ramakrishna, J. Mater. Sci., 2012, 47, 3272-3281.

43 X. Hu, S. Liu, G. Zhou, Y. Huang, Z. Xie and X. Jing, J. Controlled Release, 2014, 185, 12-21.

44 L. Zhang, K. Wang, Q. Zhao, W. T. Zheng, Z. H. Wang, S. F. Wang and D. L. Kong, Sci. China: Chem., 2010, 53, 528-534.

45 A. Szentivanyi, T. Chakradeo, H. Zernetsch and B. Glasmacher, Adv. Drug Delivery Rev., 2011, 63, 209-220.

46 Y. Fu and W. J. Kao, Expert Opin. Drug Delivery, 2010, 7, 429444.

47 J. Zeng, L. Yang, Q. Liang, X. Zhang, H. Guan, X. Xu, X. Chen and X. Jing, J. Controlled Release, 2005, 105, 43-51.

48 M. Zamani, M. P. Prabhakaran and S. Ramakrishna, Int. J. Nanomed., 2013, 8, 2997-3017.

49 T. J. Sill and H. A. von Recum, Biomaterials, 2008, 29, 19892006.

50 V. Pillay, C. Dott, Y. E. Choonara, C. Tyagi, L. Tomar, P. Kumar, L. C. du Toit and V. M. K. Ndesendo, J. Nanomater., 2013, 2013, 1-22.

51 K. Wang, X. Chen, Y. Pan, Y. Cui, X. Zhou, D. Kong and Q. Zhao, BioMed Res. Int., 2015, 2015, 865076.

52 X.-Y. Sun, L. R. Nobles, H. G. Börner and R. J. Spontak, Macromol. Rapid Commun., 2008, 29, 1455-1460.

53 Y. Chen, S. Zhou and Q. Li, Acta Biomater., 2011, 7, 11401149.

54 J. Zeng, X. Chen, Q. Liang, X. Xu and X. Jing, Macromol. Biosci., 2004, 4, 1118-1125.
55 L. L. Lao and S. S. Venkatraman, J. Biomed. Mater. Res., 2008, 87, 1-7.

56 L. Lei, S.-R. Guo, W.-L. Chen, H.-J. Rong and F. Lu, Expert Opin. Drug Delivery, 2011, 8, 813-831.

57 A. Anisimov, D. Tvorogov, A. Alitalo, V.-M. Leppänen, Y. An, E. C. Han, F. Orsenigo, E. I. Gaál, T. Holopainen, Y. J. Koh, T. Tammela, P. Korpisalo, S. Keskitalo, M. Jeltsch, S. YläHerttuala, E. Dejana, G. Y. Koh, C. Choi, P. Saharinen and K. Alitalo, Circulation, 2013, 127, 424-434.

58 S. Mitsos, K. Katsanos, E. Koletsis, G. C. Kagadis, N. Anastasiou, A. Diamantopoulos, D. Karnabatidis and D. Dougenis, Angiogenesis, 2012, 15, 1-22.

59 E. N. Unemori, N. Ferrara, E. A. Bauer and E. P. Amento, J. Cell. Physiol., 1992, 153, 557-562.

60 A. Aicher, C. Heeschen, C. Mildner-Rihm, C. Urbich, C. Ihling, K. Technau-Ihling, A. M. Zeiher and S. Dimmeler, Nat. Med., 2003, 9, 1370-1376.

61 S. S. Said, J. G. Pickering and K. Mequanint, J. Vasc. Res., 2013, 50, 35-51.

62 H. Jiang, D. Fang, B. S. Hsiao, B. Chu and W. Chen, Biomacromolecules, 2004, 5, 326-333.

63 X. Li, Y. Su, S. Liu, L. Tan, X. Mo and S. Ramakrishna, Colloids Surf., B, 2010, 75, 418-424.

64 F. Han, X. Jia, D. Dai, X. Yang, J. Zhao, Y. Zhao, Y. Fan and X. Yuan, Biomaterials, 2013, 34, 7302-7313.

65 K. Glen, N. T. Luu, E. Ross, C. D. Buckley, G. E. Rainger, S. Egginton and G. B. Nash, J. Cell. Physiol., 2012, 227, 2710-2721.

66 E. M. Domouzoglou, K. K. Naka, A. Vlahos, M. I. Papafaklis, L. K. Michalis, A. Tsatsoulis and E. Maratos-Flier, Am. J. Physiol.: Heart Circ. Physiol., 2015, 309(6), H1029-H1038.

67 P. Carmeliet and R. K. Jain, Nature, 2011, 473, 298-307.

68 R. B. Montero, X. Vial, D. T. Nguyen, S. Farhand, M. Reardon, S. M. Pham, G. Tsechpenakis and F. M. Andreopoulos, Acta Biomater., 2012, 8, 1778-1791.

69 S. Lee, G. Jin and J.-H. Jang, J. Biol. Eng., 2014, 8, 30.

70 S. S. Said, J. G. Pickering and K. Mequanint, Pharm. Res., 2014, 31, 3335-3347.

71 J. Moriya and N. Ferrara, J. Am. Coll. Cardiol., 2013, 61, E2085.

72 J. Donovan, X. Shiwen, J. Norman and D. Abraham, Fibrog. Tissue Repair, 2013, 6, 10.

73 J. Lee, J. J. Yoo, A. Atala and S. J. Lee, Biomaterials, 2012, 33, 6709-6720.

74 H. Li, C. Zhao, Z. Wang, H. Zhang, X. Yuan and D. Kong, J. Biomater. Sci., Polym. Ed., 2010, 21, 803-819.

75 A. C. Gilotti, W. Nimlamool, R. Pugh, J. B. Slee, T. C. Barthol, E. A. Miller and L. J. Lowe-Krentz, J. Cell. Physiol., 2014, 229, 2142-2152.

76 K. Nartetamrongsutt and G. G. Chase, Polymer, 2013, 54, 2166-2173.

77 X. Zong, K. Kim, D. Fang, S. Ran, B. S. Hsiao and B. Chu, Polymer, 2002, 43, 4403-4412.

78 C. Huang, S. Wang, L. Qiu, Q. Ke, W. Zhai and X. Mo, ACS Appl. Mater. Interfaces, 2013, 5, 2220-2226.

79 D. D. Swartz and S. T. Andreadis, Curr. Opin. Biotechnol., 2013, 24, 916-925. 
80 Y. Yao, J. Wang, Y. Cui, R. Xu, Z. Wang, J. Zhang, K. Wang, Y. Li, Q. Zhao and D. Kong, Acta Biomater., 2014, 10, 27392749.

81 R. Guan, X.-L. Sun, S. Hou, P. Wu and E. L. Chaikof, Bioconjugate Chem., 2004, 15, 145-151.

82 T. H. Nguyen, S.-H. Kim, C. G. Decker, D. Y. Wong, J. A. Loo and H. D. Maynard, Nat. Chem., 2013, 5, 221-227.

83 K. T. Kurpinski, J. T. Stephenson, R. R. R. Janairo, H. Lee and S. Li, Biomaterials, 2010, 31, 3536-3542.

84 N. Reddy, R. Reddy and Q. Jiang, Trends Biotechnol., 2015, 33, 362-369.

85 H. Shen, X. Hu, F. Yang, J. Bei and S. Wang, Biomaterials, 2011, 32, 3404-3412.

86 T. Okuda, K. Tominaga and S. Kidoaki, J. Controlled Release, 2010, 143, 258-264.

87 S. O. Marx, H. Totary-Jain and A. R. Marks, Circ.: Cardiovasc. Interventions, 2011, 4, 104-111.

88 A. B. Levine, D. Punihaole and T. B. Levine, Cardiology, 2012, 122, 55-68.

89 C. Napoli, G. Paolisso, A. Casamassimi, M. Al-Omran, M. Barbieri, L. Sommese, T. Infante and L. J. Ignarro, J. Am. Coll. Cardiol., 2013, 62, 89-95.

90 A. C. Mendes and A. N. Zelikin, Adv. Funct. Mater., 2014, 24, 5202-5210.

91 B. Fejerskov and A. N. Zelikin, PLoS One, 2012, 7, e49619.

92 Z. Wang, Y. Lu, K. Qin, Y. Wu, Y. Tian, J. Wang, J. Zhang, J. Hou, Y. Cui, K. Wang, J. Shen, Q. Xu, D. Kong and Q. Zhao, J. Controlled Release, 2015, 210, 179-188.

93 Q. Ji, S. Zhang, J. Zhang, Z. Wang, J. Wang, Y. Cui, L. Pang, S. Wang, D. Kong and Q. Zhao, Biomacromolecules, 2013, 14, 4099-4107.

94 Q. Zhao, J. Zhang, L. Song, Q. Ji, Y. Yao, Y. Cui, J. Shen, P. G. Wang and D. Kong, Biomaterials, 2013, 34, 8450-8458.

95 A. Andukuri, W. P. Minor, M. Kushwaha, J. M. Anderson and H.-W. Jun, Nanomedicine, 2010, 6, 289-297.

96 E. A. Lipke and J. L. West, Acta Biomater., 2005, 1, 597-606. 97 P. A. Gurbel and U. S. Tantry, JACC: Heart Failure, 2014, 2, 114.

98 C. Del Gaudio, E. Ercolani, P. Galloni, F. Santilli, S. Baiguera, L. Polizzi and A. Bianco, J. Mater. Sci.: Mater. Med., 2013, 24, 523-532.

99 P. Balakumar, Y. H. Nyo, R. Renushia, D. Raaginey, A. N. Oh, R. Varatharajan and S. A. Dhanaraj, Pharmacol. Res., 2014, 87, 144-150.

100 A. K. Sharma, D. Khanna and P. Balakumar, Int. J. Cardiol., 2014, 172, 530-532.

101 Y. B. Aldenhoff, F. H. van Der Veen, J. ter Woorst, J. Habets, L. A. Poole-Warren and L. H. Koole, J. Biomed. Mater. Res., 2001, 54, 224-233.

102 P. Punnakitikashem, D. Truong, J. U. Menon, K. T. Nguyen and Y. Hong, Acta Biomater., 2014, 10, 4618-4628.

103 S. B. Zhuplatov, T. Masaki, D. K. Blumenthal and A. K. Cheung, Basic Clin. Pharmacol. Toxicol., 2006, 99, 431-439.

104 A. E. Prota, K. Bargsten, D. Zurwerra, J. J. Field, J. F. Díaz, K.-H. Altmann and M. O. Steinmetz, Science, 2013, 339, 587-590.
105 R. A. Byrne, F.-J. Neumann, J. Mehilli, S. Pinieck, B. Wolff, K. Tiroch, S. Schulz, M. Fusaro, I. Ott, T. Ibrahim, J. Hausleiter, C. Valina, J. Pache, K.-L. Laugwitz, S. Massberg and A. Kastrati, Lancet, 2013, 381, 461-467.

106 F. Innocente, D. Mandracchia, E. Pektok, B. Nottelet, J. C. Tille, S. De Valence, G. Faggian, A. Mazzucco, A. Kalangos, R. Gurny, M. Moeller and B. H. Walpoth, Circulation, 2009, 120(11 suppl), S37-S45.

107 E. Pektok, B. Nottelet, J.-C. Tille, R. Gurny, A. Kalangos, M. Moeller and B. H. Walpoth, Circulation, 2008, 118, 2563-2570.

108 A. P. Owens and N. Mackman, Annu. Rev. Med., 2014, 65, 433-445.

109 A. Giordano, S. Romano, M. Monaco, A. Sorrentino, N. Corcione, A. L. Di Pace, P. Ferraro, G. Nappo, M. Polimeno and M. F. Romano, Am. J. Physiol.: Heart Circ. Physiol., 2012, 302, H135-H142.

110 Y. Liu, J. Wei, S. Hu and L. Hu, Am. J. Biomed. Sci., 2012, 344, 220-226.

111 C. Zheng, V. Azcutia, E. Aikawa, J.-L. Figueiredo, K. Croce, H. Sonoki, F. M. Sacks, F. W. Luscinskas and M. Aikawa, Eur. Heart J., 2013, 34, 615-624.

112 C. H. Lee, S. H. Chang, Y. H. Lin, S. J. Liu, C. J. Wang, M. Y. Hsu, K. C. Hung, Y. H. Yeh, W. J. Chen, I. C. Hsieh and M. S. Wen, Biomaterials, 2014, 35, 4417-4427.

113 T. F. Lüscher, J. Steffel, F. R. Eberli, M. Joner, G. Nakazawa, F. C. Tanner and R. Virmani, Circulation, 2007, 115, 10511058.

114 T. Palmerini, G. Biondi-Zoccai, D. Della Riva, C. Stettler, D. Sangiorgi, F. D'Ascenzo, T. Kimura, C. Briguori, M. Sabatè, H.-S. Kim, A. De Waha, E. Kedhi, P. C. Smits, C. Kaiser, G. Sardella, A. Marullo, A. J. Kirtane, M. B. Leon and G. W. Stone, Lancet, 2012, 379, 1393-1402.

115 S. Betha, B. Pamula Reddy, M. Mohan Varma, D. Basava Raju and V. Ramana Murthy Kolapalli, J. Pharm. Invest., 2014, 45, 13-22.

116 N. M. Vacanti, H. Cheng, P. S. Hill, J. D. T. Guerreiro, T. T. Dang, M. Ma, S. Watson, N. S. Hwang, R. Langer and D. G. Anderson, Biomacromolecules, 2012, 13, 3031-3038.

117 W. Chen, D. Li, A. EI-Shanshory, M. El-Newehy, H. A. EIHamshary, S. S. Al-Deyab, C. He and X. Mo, Colloids Surf., $B, 2015,126,561-568$.

118 K. Liu, C. Lee, Y. Wang and S. Liu, Int. J. Nanomed., 2015, 885-891.

119 K.-S. Liu, S.-J. Liu, H.-Y. Chen, Y.-K. Huang, Y.-J. Peng, R.-C. Wu and S. W.-N. Ueng, Chest, 2012, 141, 1197-1202.

120 G. C. Inaraja Pérez, A. Rodríguez Morata, J. P. Reyes Ortega, R. Gómez Medialdea and P. Cabezudo García, Ann. Vasc. Surg., 2015, 29, 1018.

121 S. Singh, A. Sharma, M. Minz, D. Kenwar and S. Kumar, J. Vasc. Access, 2015, 16, e65.

122 J. Kluin, H. Talacua, A. I. P. M. Smits, M. Y. Emmert, M. C. P. Brugmans, E. S. Fioretta, P. E. Dijkman, S. H. M. Söntjens, R. Duijvelshoff, S. Dekker, M. W. J. T. Janssen-van den Broek, V. Lintas, A. Vink, S. P. Hoerstrup, H. M. Janssen, P. Y. W. Dankers, 
F. P. T. Baaijens and C. V. C. Bouten, Biomaterials, 2017, 125, 101-117.

123 A. H. Zisch, M. P. Lutolf, M. Ehrbar, G. P. Raeber, S. C. Rizzi, N. Davies, H. Schmökel, D. Bezuidenhout, V. Djonov, P. Zilla and J. A. Hubbell, FASEB J., 2003, 17, 2260-2262.

124 M. Chen, Y.-F. Li and F. Besenbacher, Adv. Healthcare Mater., 2014, 3, 1721-1732.

125 M. Qi, X. Li, Y. Yang and S. Zhou, Eur. J. Pharm. Biopharm., 2008, 70, 445-452.

126 Z. Yuan, J. Zhao, W. Zhu, Z. Yang, B. Li, H. Yang, Q. Zheng and W. Cui, Biomater. Sci., 2014, 2, 502-511.

127 J. C. Garbern, E. Minami, P. S. Stayton and C. E. Murry, Biomaterials, 2011, 32, 2407-2416.

128 F. Song, X.-L. Wang and Y.-Z. Wang, Colloids Surf., B, 2011, 88, 749-754.

129 H. Yuan, B. Li, K. Liang, X. Lou and Y. Zhang, Biomed. Mater., 2014, 9, 55001.

130 X. J. Loh, P. Peh, S. Liao, C. Sng and J. Li, J. Controlled Release, 2010, 143, 175-182.

131 L. Weng and J. Xie, Curr. Pharm. Des., 2015, 21, 1944-1959.

132 D. Roy, J. N. Cambre and B. S. Sumerlin, Prog. Polym. Sci., 2010, 35, 278-301.
133 G. M. Xiong, S. Yuan, J. K. Wang, A. T. Do, N. S. Tan, K. S. Yeo and C. Choong, Acta Biomater., 2015, 23, 240-249.

134 N. A. Raof, W. B. Pratt, L. Pradhan-Nabzdyk, F. W. LoGerfo, S. G. Pathan, D. W. Nelson and M. D. Phaneuf, in 2014 40th Annual Northeast Bioengineering Conference (NEBEC), IEEE, 2014, pp. 1-2.

135 C. S. Nabzdyk, M. C. Chun, H. S. Oliver-Allen, S. G. Pathan, M. D. Phaneuf, J.-O. You, L. K. Pradhan-Nabzdyk and F. W. LoGerfo, Biomaterials, 2014, 35, 3071-3079.

136 M. Enayati, M.-W. Chang, F. Bragman, M. Edirisinghe and E. Stride, Colloids Surf., A, 2011, 382, 154-164.

137 X. Guo, T. Xia, H. Wang, F. Chen, R. Cheng, X. Luo and X. Li, Pharm. Res., 2014, 31, 874-886.

138 L. Yu, Y. Feng, Q. Li, X. Hao, W. Liu, W. Zhou, C. Shi, X. Ren and W. Zhang, React. Funct. Polym., 2015, 91-92, 19-27.

139 F. Chen, H. Wan, T. Xia, X. Guo, H. Wang, Y. Liu and X. Li, Eur. J. Pharm. Biopharm., 2013, 85, 699-710.

140 X. Shi, G. Chen, L.-W. Guo, Y. Si, M. Zhu, S. Pilla, B. Liu, S. Gong and K. C. Kent, PLoS One, 2014, 9, e89227.

141 H. Wang, Y. Zhang, T. Xia, W. Wei, F. Chen, X. Guo and X. Li, Mol. Pharm., 2013, 10, 2394-2403. 